\title{
Electron mobility in oxide heterostructures
}

\section{Topical review}

\section{Trier, F.; Christensen, Dennis Valbjørn; Pryds, Nini}

Published in:

Journal of Physics D: Applied Physics

Link to article, DOI:

10.1088/1361-6463/aac9aa

Publication date:

2018

Document Version

Peer reviewed version

Link back to DTU Orbit

Citation (APA):

Trier, F., Christensen, D. V., \& Pryds, N. (2018). Electron mobility in oxide heterostructures: Topical review. Journal of Physics D: Applied Physics, 51(29), [293002]. https://doi.org/10.1088/1361-6463/aac9aa

\section{General rights}

Copyright and moral rights for the publications made accessible in the public portal are retained by the authors and/or other copyright owners and it is a condition of accessing publications that users recognise and abide by the legal requirements associated with these rights.

- Users may download and print one copy of any publication from the public portal for the purpose of private study or research.

- You may not further distribute the material or use it for any profit-making activity or commercial gain

- You may freely distribute the URL identifying the publication in the public portal

If you believe that this document breaches copyright please contact us providing details, and we will remove access to the work immediately and investigate your claim. 


\title{
Electron Mobility in Oxide Heterostructures
}

\author{
F. Trier ${ }^{1, *}$, D. V. Christensen ${ }^{2, *}$ and N. Pryds ${ }^{2}$
}

${ }^{1}$ Unité Mixte de Physique CNRS, Thales, Université Paris-Sud, Université Paris-Saclay, 91767 Palaiseau, France.

2 Department of Energy Conversion and Storage, Technical University of Denmark, Ris $\varnothing$ Campus, DK-4000 Roskilde, Denmark

${ }^{*}$ Equally contributing authors

\begin{abstract}
Next-generation integrated circuit devices based on transition-metal-oxides are expected to boast a variety of extraordinary properties, such as superconductivity, transparency in the visible range, thermoelectricity, giant ionic conductivity and ferromagnetism. However, the realisation of this socalled oxide electronics as well as the study of their unconventional physics is stalled by inferior carrier mobilities compared to conventional semiconductor materials. Over the past 10 years, bulk conducting oxides and oxide heterostructures with superior carrier mobilities have nonetheless seen significant progress. This progress is signifying the approaching era of oxide-based electronic circuits along with novel solid-state phenomena originating from the combination of hybridized oxygen $p$ orbitals, transition-metal $d$ orbitals and electronic correlations. Here, we review the recent advancements and results on high mobility oxide heterostructures based on $\mathrm{SrTiO}_{3}$ and $\mathrm{ZnO}$ as well as other prominent oxides.
\end{abstract}

\section{Section 1: Introduction}

The race for achieving high carrier mobility in semiconductor materials has proved beneficial from both a scientific and technological perspective. It has led to electron mobilities exceeding $35,000,000 \mathrm{~cm}^{2} / \mathrm{Vs}$ in $\mathrm{Al}_{\mathrm{x}} \mathrm{Ga}_{1-\mathrm{x}} \mathrm{As}-$ based material systems [1], the realization of quantum phenomena such as integer and fractional quantum Hall effect $[2,3]$ and new concepts for achieving pure materials with high carrier mobilities [4]. High carrier mobilities are particularly beneficial for: i) the study of quantum phenomena where extended mean free paths are required. ii) an increased control over detrimental scattering mechanisms. iii) applications requiring fast electronic responses (e.g. high-frequency devices). iv) achieving lowresistance materials without increasing the carrier density, which is advantageous for, e.g., thermoelectric materials and transparent conductors. Whereas the development of high-mobility silicon and III-V semiconductors in many cases is very mature, appealing functionalities can be obtained by considering materials beyond these. Some of the new promising materials for these high mobility electronic applications are the oxides and oxide heterostructures.

\section{1 - Why oxides?}

Heterostructures based on transition-metal-oxides (TMOs) exhibit a variety of extraordinary properties, such as superconductivity, transparency in the visible range, thermoelectricity, giant ionic conductivity and ferromagnetism. The electronic properties of transitionmetal oxides are determined by a delicate balance between hybridized oxygen $p$ orbitals, transition-metal $d$ orbitals and electronic correlations. The interfaces based on TMOs can therefore lead to unconventional physics and find use in the present and coming generations of electronic devices. Transparent conducting oxides are for example heavily used in flat panel displays and solar cells.

The interest in these transition-metal oxides has motivated the research in epitaxial growth of layered oxides such as oxide heterostructures. This line of research seeks, in part, to emulate the achievements of previous generations of semiconductor materials researchers who over the past few decades have learned to engineer the electronic properties of epitaxial grown semiconductor materials by exploiting e.g. epitaxial strain and modulation doping. Because of the wider range of phenomena, the implications for physics and technology of advances in transition-metal oxides are likely to be large. The worldwide race between crystal growers from major research laboratories to continuously improve the growth of oxide interfaces has led to high electron mobilities. One of the most extensively studied metal oxide heterointerface systems is the $\mathrm{LaAlO}_{3} / \mathrm{SrTiO}_{3}$ (LAO/STO) interface [5]. The initial mobility value of $10^{3}$ $\mathrm{cm}^{2} / \mathrm{V}$ s of this system was surpassed by replacing the perovskite $\mathrm{LaAlO}_{3}$ with spinel structured $\mathrm{\gamma}-\mathrm{Al}_{2} \mathrm{O}_{3}$, which resulted in a record-high electron mobility greater than $10^{5} \mathrm{~cm}^{2} / \mathrm{Vs}$ at $2 \mathrm{~K}$ [6]. Another system that is well studied is the $\mathrm{ZnO} / \mathrm{ZnMgO}$ material system, which shows extremely high mobilities exceeding $10^{6} \mathrm{~cm}^{2} / \mathrm{Vs}$ at $2 \mathrm{~K}$ [7]. These electron mobilities were made possible by improving the quality of the epitaxial oxide thin films prepared with state-of-the-art deposition techniques such as oxide molecular beam epitaxy (MBE) or pulsed laser deposition (PLD), which allow the growth of heterostructures with very sharp interfaces. By fabricating and understanding the high mobility in oxides, we may be able to produce high-quality electrical components with the added functionalities that oxides exhibit. 
In this paper, we review the advancements and recent results made in high mobility oxide heterostructure materials. First, we describe the relevant scattering mechanisms and general pathways used to enhance the mobility in Section 2. In Section 3 we review the electron mobility in threedimensional conducting $\mathrm{SrTiO}_{3}$ (STO) while Section 4 presents a detailed discussion on the high mobility obtained in confined electron gases of representative STO-based systems. We then review the electron mobility of three-dimensional conducting $\mathrm{ZnO}$ in Section 5 whereas the subsequent Section 6 covers the development in high-mobility $\mathrm{Mg}_{\mathrm{x}} \mathrm{Zn}_{1-\mathrm{x}} \mathrm{O} / \mathrm{ZnO}$ heterostructures. Lastly, in Section 7 we provide a brief overview of the literature concerning mobility in oxides beyond STO and ZnO.

\section{Section 2: Scattering mechanism \& pathways to enhance mobility}

Improving the mobility of any conductor is of importance for fundamental understanding of solid-state physics but also for applications. Therefore, understanding the different carrier scattering mechanisms that limits the mobility in functional materials such as oxides is similarly important.

When attempting to understand electronic transport and optical properties in oxides, one essentially aims at accessing the eigenfunction and total energy of the electrons residing in the particular oxide. The universally employed method for accessing this information is the so-called effective mass approximation where electrons essentially are treated as free electrons with a modified mass, $m^{\star}$.

When an electric field $(E)$ is applied to the oxide, charge carriers will accelerate. The acceleration is counteracted by elastic or inelastic scattering events, and the charge carriers end up travelling with an average drift velocity $\left(v_{d}\right)$. The electron mobility $(\mu)$ is defined as the ratio between the drift velocity and electric field:

$$
\mu=v_{d} / E
$$

A large carrier mobility can be obtained by increasing the scattering time $(\tau)$ or decreasing the effective mass $\left(m^{*}\right)$ :

$$
\mu=\frac{e \tau}{m^{*}}
$$

The scattering time can vary greatly from oxide to oxide depending on e.g. the defect concentration, carrier concentration and phonon.

The electron or hole mobility is typically measured experimentally using the Hall effect. As charges move in an out-of-plane magnetic field, the current $(I)$ will deflect and form a transverse Hall voltage $\left(V_{H}\right)$. The Hall voltage can be related to the carrier density $(n)$ and mobility $(\mu)$ by

$$
R_{H}=\frac{d\left(V_{H} / I\right)}{d B}=-\frac{1}{n e}
$$

and

$$
\mu=\frac{R_{H}}{R}
$$

with $R$ being the resistivity of the material. This mobility is known as the Hall mobility and is importantly used as a figure-of-merit between different oxide conductors. In this case, $\tau$ in Eq. 2 describes the momentum relaxation time $\left(\tau_{H}\right)$ and corresponds to the average time it takes for the momentum of a charge carrier to reduce to $37 \%$ due to scattering events. Another characteristic mobility of materials is the quantum mobility in which the relevant time scale $\left(\tau_{q}\right)$ relates to the time it takes loose quantum coherence by the scattering events. This is typically measured using Shubnikov-de Haas oscillations. The quantum mobility may differ significantly from the Hall mobility as established in heterostructures based on GaAs [8], STO $[9,10]$ and ZnO [11].

Table 1 shows examples of $m^{\star}$, Hall mobility and quantum mobility at $T<$ $2 \mathrm{~K}$ when electrons reside in a heterostructure for two important oxides that will be the focus of this review: STO and ZnO.

\begin{tabular}{|l|c|c|c|}
\hline $\begin{array}{l}\text { Material } \\
\text { system }\end{array}$ & $\boldsymbol{m}^{\star}$ & $\boldsymbol{\mu}$, Hall (2 K) & $\boldsymbol{\mu}$, quantum (20 mK) \\
\hline $\mathrm{STO}[6]$ & $1 m_{e}$ & $1.4 \times 10^{5} \mathrm{~cm}^{2} / \mathrm{Vs}$ & $7.2 \times 10^{3} \mathrm{~cm}^{2} / \mathrm{Vs}$ \\
\hline $\mathrm{ZnO}[7]$ & $0.3 m_{e}$ & $1.2 \times 10^{6} \mathrm{~cm}^{2} / \mathrm{Vs}$ & $4.8 \times 10^{4} \mathrm{~cm}^{2} / \mathrm{Vs}$ \\
\hline
\end{tabular}

Table 1 - Values of the effective mass $\left(m^{\star}\right)$, Hall mobility $(\mu$, Hall) and quantum mobility ( $\mu$, quantum) for electrons residing in state-of-the-art STO and ZnO heterostructures. Note that the quantum and Hall mobility values reported here were obtained from two different STO-based and ZnO-based samples.

The charge carriers undergo scattering from one or multiple sources and the total mobility $(\mu)$ is typically assumed to be composed of the individual scattering contributions $\left(\mu_{i}\right)$ according to the Matthiesen rule:

$$
\mu^{-1}=\sum_{i} \mu_{i}^{-1}
$$

In the following we will introduce some important scattering mechanisms for oxides. All subsequent equations are in their generalized form (i.e. not material specific) and describe the case for three-dimensional conducting structures. Here it is important to note that alternative expressions exist for confined electronic systems such as two-dimensional electron gases, but these will not be covered here. Additional information on various scattering mechanisms, and mobility expressions for electronic systems with reduced dimensionality can be found in, e.g., $[12,13]$.

\section{1 - lonized Donor and Impurity Scattering}

In many cases the dominant scattering mechanism at low temperatures is scattering off ionized point defects or defect clusters. This includes ionized donors that are intentionally introduced to the materials as well as ionized impurities that unintentionally are present in oxides often in high concentrations.

For oxides in the low carrier density regime where the contribution from ionized impurity scattering is largest, it is possible to express the electron mobility as follows:

$$
\mu=\frac{3 \pi}{2} \frac{e}{\hbar} a_{B}^{2} \frac{n}{N_{S} \ln \left(\pi k_{F} a_{B}\right)}
$$

where $a_{B}$ is the effective Bohr radius given by $a_{B}=4 \pi \varepsilon_{r} \varepsilon_{0} \hbar^{2} / m^{\star} e^{2}, n$ is the carrier density and $N_{S}$ is the concentration of ionized impurities.

At large carrier concentrations, $n$ will become comparable with $N_{s}$. Beyond this point, the concentration of ionized donors in the oxide will exceed the concentration of ionized impurities and most electrons will scatter on donors. Thus, the mobility in the large carrier density regime is given by: 


$$
\mu=\frac{e}{\hbar} \frac{1}{n a^{2} k_{F}}
$$

where $a$ is the lattice size of the given oxide. To reduce the effect of ionized donor scattering, one can employ delta doping or modulation doping strategies $[14,15]$. In delta doping, the donors are positioned in a thin sheet whereas the charge carriers extend beyond this, and thus part of the charge carriers reside in parts of the oxide without charged donors. In modulation doping, the electrons and donors are spatially separated so that the interaction is only through long-range Coulomb attraction.

If the defects remain neutral, the scattering cross-section is much smaller than the ionized defects and therefore generally less detrimental to the overall mobility.

\section{2 - Electron-phonon interaction}

As the temperature increases, more phonon modes become populated and the interaction between charge carriers and lattice vibrations becomes gradually more important for determining the carrier mobility. Lattice vibrations are particularly important in oxides where the high electronegativity of oxygen creates polar bonds leading to polaron formation and oscillating electric fields that may severely affect the charge flow:

\subsection{1 - Polaron formation}

As the charged carriers move through the ionic oxide, charge-lattice interactions deform the lattice. If the interaction is sufficiently strong, the charge carriers can no longer be considered to be freely moving, but rather move in the lattice as a heavier polaron. The effect of polaron formation is generally described through a mass renormalization where the band effective mass $\left(m_{b}\right)$ is replaced with a polaron effective mass $\left(m_{p}\right)$. The increased mass of the polarons leads to a lowering of the mobility as it will be presented in Section 4.2.1 for the case of STO-based structures.

The mass enhancement can be circumvented by lowering the electronphonon coupling $(\alpha)$ according to $m_{p}=m_{b}(1+\alpha / 6)$ and $m_{p}=m_{b}(1+$ $\left.(\pi / 8) \alpha+0.1272 \alpha^{2}\right)$, which is valid for three- and two-dimensionally confined carriers, respectively, with Fröhlich interactions to a threedimensional lattice [16],[17].

\subsection{2 - Acoustic phonon scattering}

The coherent motion of acoustic phonons typically scatter charge carriers by locally offsetting the bands. When considering e.g. $n$-type oxides, the acoustic phonon modes change the interatomic distances and lead to local changes in the conduction band that scatters the electrons. The contribution to the mobility $\left(\mu_{A C}^{3 D}\right)$ is given by $[12,18]$ :

$$
\mu_{A C}^{3 D}=\frac{2 e \hbar \rho v_{S}^{2}}{3 \pi n_{3 D} m^{\star} a_{C}^{2}} \ln \left(1+\exp \left(E_{F} / k_{B} T\right)\right)
$$

where $\rho$ is the crystal density, $v_{S}$ is the velocity of sound, $n_{3 D}$ is the threedimensional carrier density, $E_{F}$ is the Fermi energy, $a_{c}$ is the conduction band deformation potential, $k_{B}$ is Boltzmann constant and $T$ is the temperature.

Acoustic phonon scattering is reported to be the dominant scattering mechanism for e.g. $p$-type $\mathrm{ZnO}$ from $T=10-300 \mathrm{~K}$ [19]. The acoustic phonon scattering can generally be counteracted by decreasing the temperature, varying the carrier density and modifying the phonon modes.

\subsection{3 - Optical phonon scattering}

When the longitudinal optical (LO) phonon modes of an ionic lattice are excited, macroscopic electric fields are created that scatter the charge carriers. In the weak/intermediate coupling regime where $\alpha<6$, Low and Pines derived an expression for the mobility used frequently for threedimensional conducting STO (as well as two-dimensionally confined carriers in STO-based structures) [16]:

$$
\mu_{L O}=\frac{\hbar}{2 \alpha \hbar \omega_{L O}} \frac{e}{m_{p}}\left(\frac{m_{p}}{m_{b}}\right)^{3} f(\alpha) \exp \left(\frac{\hbar \omega_{L O}}{k_{B} T}\right)
$$

where $\omega_{L O}$ is the angular frequency of the longitudinal optical phonon mode and $f(\alpha)$ is a monotonic function of $\alpha$ that varies slowly from 1 to 1.35 as $\alpha$ increases from 0 to 6 [16]. Other expressions for longitudinal optical phonon scattering have also been derived [12].

Transverse optical (TO) phonon modes, however, create no net macroscopic electric field, and they interact with the electrons through deformation of the bands. The associated mobility contribution $\left(\mu_{\mathrm{TO}}^{3 D}\right)$ can be written as $[12,18]$

$$
\mu_{\mathrm{TO}}^{3 D}=\frac{\sqrt{2} \pi e \hbar^{3} \rho v_{S}^{2}}{\left(m^{\star}\right)^{5 / 2} \omega_{T O} d_{0}^{2}} \frac{\exp \left(\hbar \omega_{T O} / k_{B} T\right)-1}{\sqrt{E_{F}+\hbar \omega_{T O}}}
$$

where $\omega_{T O}$ is the angular frequency of the transverse optical phonon mode and $d_{0}$ is the optical phonon deformation potential.

The general pathways to decrease the influence of optical phonon scattering include lowering the temperature, decreasing the electronphonon coupling/deformation potential and modifying the phonon modes. The latter can be done e.g. using strain, elemental substitution with lighter or heavier atoms or using interfaces or thin films to break the lattice symmetry [12].

\subsubsection{Piezoelectric scattering}

Crystals without inversion symmetry can be piezoelectric active, and here the acoustic phonon modes may lead to changes in the electric fields that can scatter charge carriers strongly. The mobility in e.g. $p$-type $\mathrm{ZnO}$ is significantly limited by piezoelectric scattering $[19,20]$.

\section{3 - Electron-electron scattering}

Electron-electron interactions often play an important role in determining the properties of oxides. This is particularly the case for correlated transition metal oxides with the conduction band derived from $d$-orbitals. The electron-electron interaction may also severely affect the carrier mobility. The Hall mobility is only sensitive to changes in the total carrier momentum. This leaves the Hall mobility insensitive to regular electronelectron collisions where the momentum is conserved. However, some electron-electron collisions involve the lattice such as Umklapp scattering events and collisions that promote electrons to another band with a different effective mass. The contribution from electron-electron interactions $\left(\mu_{e e}\right)$ is usually described by

$$
\mu_{e e}=A T^{-2}
$$

where, $A$ is the temperature coefficient and $T$ is the temperature. For STO, this contribution is likely to be the dominant scattering mechanism for temperatures between $5 \mathrm{~K}<T<150 \mathrm{~K}$ [21].

\section{4 - Interface roughness scattering}

For many devices it is crucial to confine the charge carriers to two or fewer dimensions in order to effectively tune the device properties with a gate. This requires a broken symmetry, which is typically created by making a heterostructure or exploiting the surface of the material. In both cases, the broken lattice symmetry inherently forms imperfections and defects, which serves as scattering sites for the charge carriers. The common procedure to reduce the interface roughness scattering is to lattice match the materials used to form the heterostructure. For additional details on interface roughness scattering in e.g. STO, see reference [22].

\section{5 - Additional scattering mechanisms}


There are several other scattering mechanisms that are not described in detail here. These includes (but are not limited to) scattering with extended lattice imperfections such as grain boundaries, domain walls and dislocations [12]. Spin-related scattering mechanisms may also be of high importance for materials exhibiting e.g. Kondo effect and colossal magnetoresistance [12].

\section{Section 3: Electron mobility in bulk $\mathrm{SrTiO}_{3}$}

Electronic transport in bulk conducting $\mathrm{SrTiO}_{3}$ (STO) have been studied for more than half a century. Since the first papers appeared in the early $1960 \mathrm{~s}$ $[23,24]$, much effort has been spent in understanding how electrons are transported through STO. STO displays both metallic behaviour and superconductivity for a wide range of charge carrier densities remarkably even down to very dilute values [25]. The charge carriers in STO originate either from intentional donors or from a reduction of the STO crystal - i.e. formation of oxygen vacancies through vacuum annealing. Much of the exciting physics in STO arise due to the empty $3 d$ shell of titanium. Due to the crystal field splitting, the $3 d$ levels split into the twofold and threefold degenerate $e_{g}$ and $t_{2 g}$ levels, respectively. When STO is doped, electrons populate the $t_{2 g}$ levels, which lead to a bulk $n$-type metallic conductivity of STO [26].

When STO display metallic behaviour, the carrier density changes very little as the temperature is reduced from room temperature to cryogenic temperatures. However, as the dielectric constant of STO, and consequently the degree of electrostatic screening, undergoes dramatic changes in this temperature interval, the electron mobility in STO is profoundly susceptible to temperature changes (see Figure 1). Through this change in electrostatic screening, the electron mobility can increase by almost a staggering four orders of magnitude as the temperature is reduced from $300 \mathrm{~K}$ to $2 \mathrm{~K}$, reaching values well in excess of $10,000 \mathrm{~cm}^{2} / \mathrm{Vs}$ (see Figure 1). This exciting behaviour has fostered a substantial and ongoing effort in trying to understand the underlying solid-state physics of bulk conducting STO. The physical properties of STO are given in Table 2.

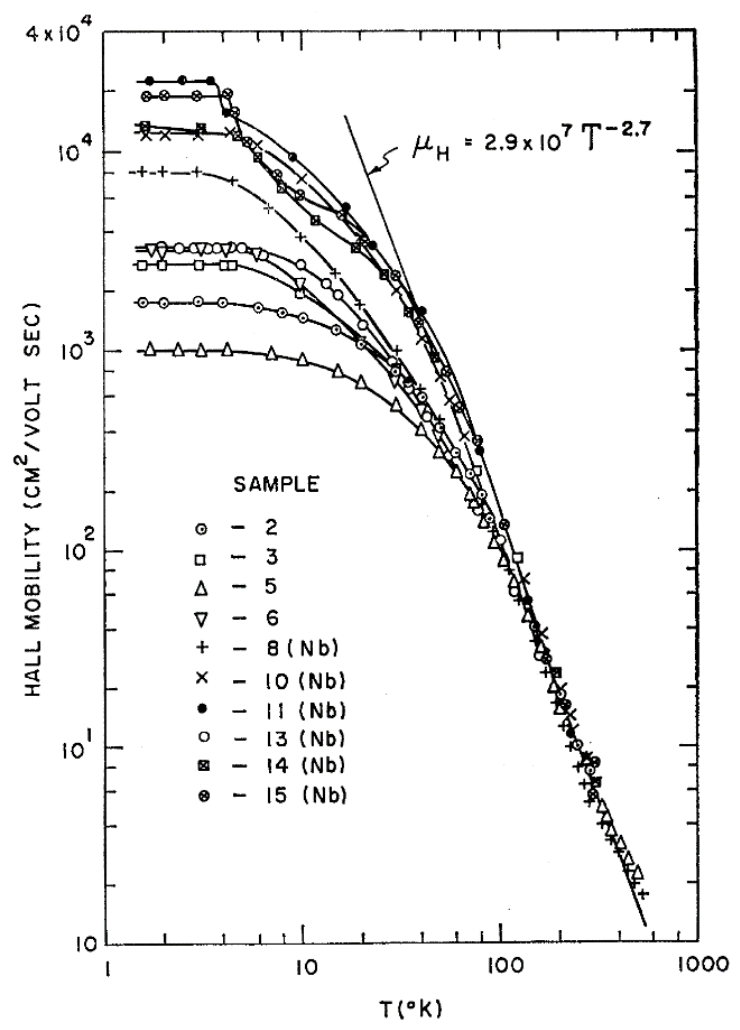

Figure 1 - Initial studies of bulk conducting STO uncovered a large temperature dependence of the electron mobility. Reprinted figure with permission from [23]. Copyright 1967 by the American Physical Society.

\begin{tabular}{|l|c|c|}
\hline Physical property & Value & Ref. \\
\hline Molar mass & $183.49 \mathrm{~g} / \mathrm{mol}$ & PT \\
\hline Appearance & White, opaque crystals & PT \\
\hline Density & $5.11 \mathrm{~kg} / \mathrm{m}^{3}$ & PT \\
\hline Melting point & $2080^{\circ} \mathrm{C}$ & PT \\
\hline Boiling point & 2.394 & PT \\
\hline Refractive index & PT \\
\hline Direct and wide band gap & $\begin{array}{c}\text { Indirect } / \text { direct band gap of } \\
3.26 \mathrm{eV} / 3.75 \mathrm{eV} \text { at } 2 \mathrm{~K}\end{array}$ & {$[27,28]$} \\
\hline Large exciton binding energy & $220 \mathrm{meV}$ & {$[29]$} \\
\hline Piezoelectric constants & $14 \mathrm{C} / \mathrm{m}^{2}\left(\mathrm{e}_{33}\right),-0.5 \mathrm{C} / \mathrm{m}^{2}\left(\mathrm{e}_{31}\right)$ & {$[30]$} \\
\hline Effective mass & $1 \mathrm{me}$ & {$[6]$} \\
\hline $\begin{array}{l}\text { High-frequency dielectric } \\
\text { constant }\end{array}$ & 100 at $2 \mathrm{~K}$ & {$[31]$} \\
\hline Static dielectric constant & 20000 at $2 \mathrm{~K}$ & {$[32]$} \\
\hline Lattice constant & $\mathrm{a}=3.904 \AA$ at $2 \mathrm{~K}$ & {$[33]$} \\
\hline Sound velocity & $7.9 \times 10^{3} \mathrm{~m} / \mathrm{s}$ at $300 \mathrm{~K}$ & {$[34]$} \\
\hline
\end{tabular}

Table 2 - The physical parameters of STO adopted from the literature or the periodic table (PT).

\section{1 - Temperature dependence of the mobility}

A large number of different scattering mechanisms have been used to try to explain the mobility of STO as a function of the temperature, which vary greatly (see Figure 1). In general, the temperatures are divided into three regimes: At low temperatures (typically $T<10 \mathrm{~K}$ ), scattering with ionized impurities and donors in STO dominate charge carrier transport. This temperature regime will be discussed in Subsection 3.3. At intermediate 
temperatures (typically $10 \mathrm{~K}<T<150 \mathrm{~K}$ ), a $\mu \propto T^{-n}$ behaviour with $n \sim 2$ is observed and generally ascribed to electron-electron interactions [21,35-37] (see Figure 2), although other explanations have also been proposed based on temperature dependent polaron [38] or dielectric [39] properties and scattering with acoustic [18], transverse optical [18] or longitudinal optical phonons [39]. The detailed understanding of the scattering mechanism in this temperature regime remains elusive, and Mikheev et al. indeed recently showed that the scattering rate is independent on the carrier density in contrast to what is expected from Landau Fermi liquid theory [35]. Lastly, as it will be discussed in the next subsection, the dominant electron scattering contribution close to room temperature $(T>150 \mathrm{~K}$ ) is generally attributed to longitudinal optical (LO) phonons $[21,23,24]$. However, in particular for high carrier densities, the electron-electron interaction may dominate all the way up to room temperature [21].

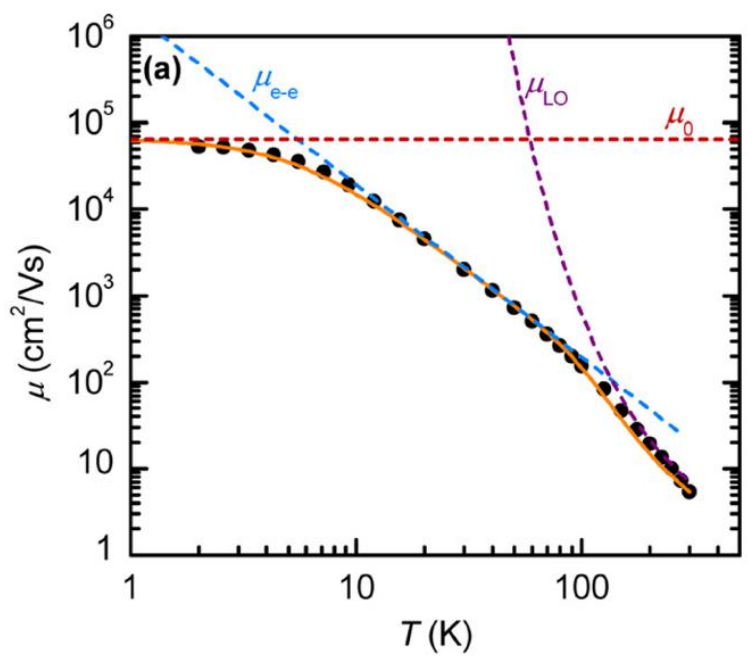

Figure 2 - Comparison of scattering model and data for electron mobility of La-doped $\mathrm{SrTiO}_{3}$ thin films. Here, the scattering model consider electronelectron interactions as a significant scattering contribution to electron mobility at temperatures between $T=10-150 \mathrm{~K}$. Reprinted from reference [21], with the permission of AIP Publishing.

\section{2 - Electron mobility at room temperature}

Unlike most conventional materials, the room temperature electron mobility of STO is almost independent with the carrier density. Specifically, the electron mobility remains between $\sim 1-8 \mathrm{~cm}^{2} / \mathrm{Vs}$ for carrier densities spanning several orders of magnitude between $10^{16}-5 \times 10^{20} \mathrm{~cm}^{-3}$ (see Figure 3). Note that only samples with $n_{3 D}>2 \times 10^{16} \mathrm{~cm}^{-3}$ presented in Figure 3 showed metallic behaviour down to $T=2 \mathrm{~K}$, whereas samples with $n_{3 D}<$ $2 \times 10^{16} \mathrm{~cm}^{-3}$ showed a resistance upturn at some point while cooling from room temperature.

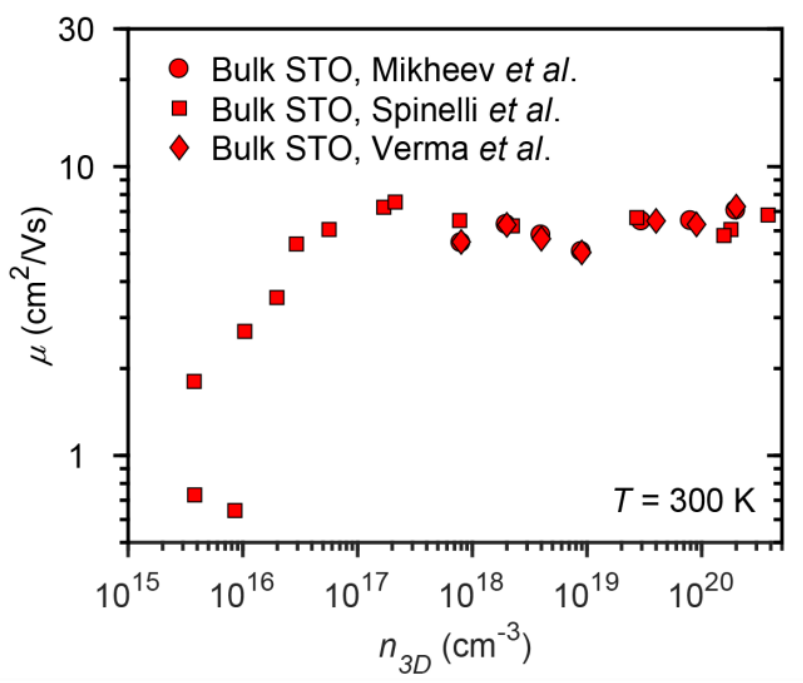

Figure 3 - The room-temperature electron mobility of bulk conducting $\mathrm{SrTiO}_{3}$ change little over several orders of magnitude charge carrier densities. The data is obtained from Verma et al. [18], Mikheev et al. [21], Spinelli et al. [40].

As mentioned above, LO phonons are thought to be one of the dominant contributions to room temperature electron scattering in STO. The effect of this contribution is reduced in most materials when the charge carrier density is increased. In other words, more charge carriers correspond to less scattering due to an increased effective screening of the phonons. However, for small charge carrier densities, the electron mobility of STO remains essentially unaltered compared to the situation at large carrier densities (see Figure 3). Mikheev et al. suggested that this unconventional behaviour of the electron mobility with carrier density was due to the presence of a significant electron-electron scattering contribution [21]. The effect of this contribution should scale directly with the charge carrier density, i.e. with less charge carriers corresponding to less scattering. This electron-electron scattering mechanism will hence compete with the scattering contribution due to LO phonons. Interestingly, these two effects should overall cancel with each other leading to the observed constant electron mobility with charge carrier density. Mikheev et al. estimated the crossover carrier density where the two contributions are equal to be around $n_{3 D} \sim 6 \times 10^{18} \mathrm{~cm}^{-3}$ (see Figure 4). 


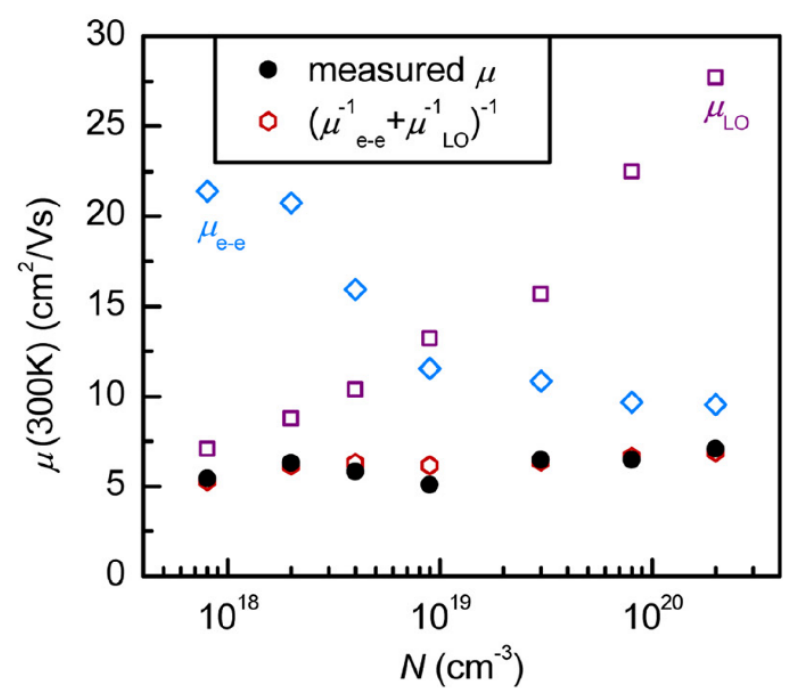

level [41]. This means that for $n_{3 D} \ll N_{s}$, electrons in STO will primarily scatter on ionized background impurities and consequently represent the intrinsic upper limit of electron mobility in STO. On the other hand, when $n_{3 D} \gg N_{s}$, the low-temperature electron mobility is instead limited by scattering on the intentionally added ionized donors. These different scattering regimes and the corresponding overall electron mobility in STO is given according to as described in Subsection 2.1 and Equations 6 and 7 . Using the Matthiessen additive rule to the different contributions, it is possible to express the overall electron mobility for any given charge carrier density. This overall mobility obtained by combination of the different scattering contributions can then be calculated for carrier densities between $1 \times 10^{17}-5 \times 10^{20} \mathrm{~cm}^{-3}$ and is shown in Figure 5 .

Figure 4 - The measured room-temperature electron mobility was proposed by Mikheev et al. to arise from an effective cancelling of the electron-electron and LO scattering contributions. Reprinted from reference [21], with the permission of AIP Publishing.

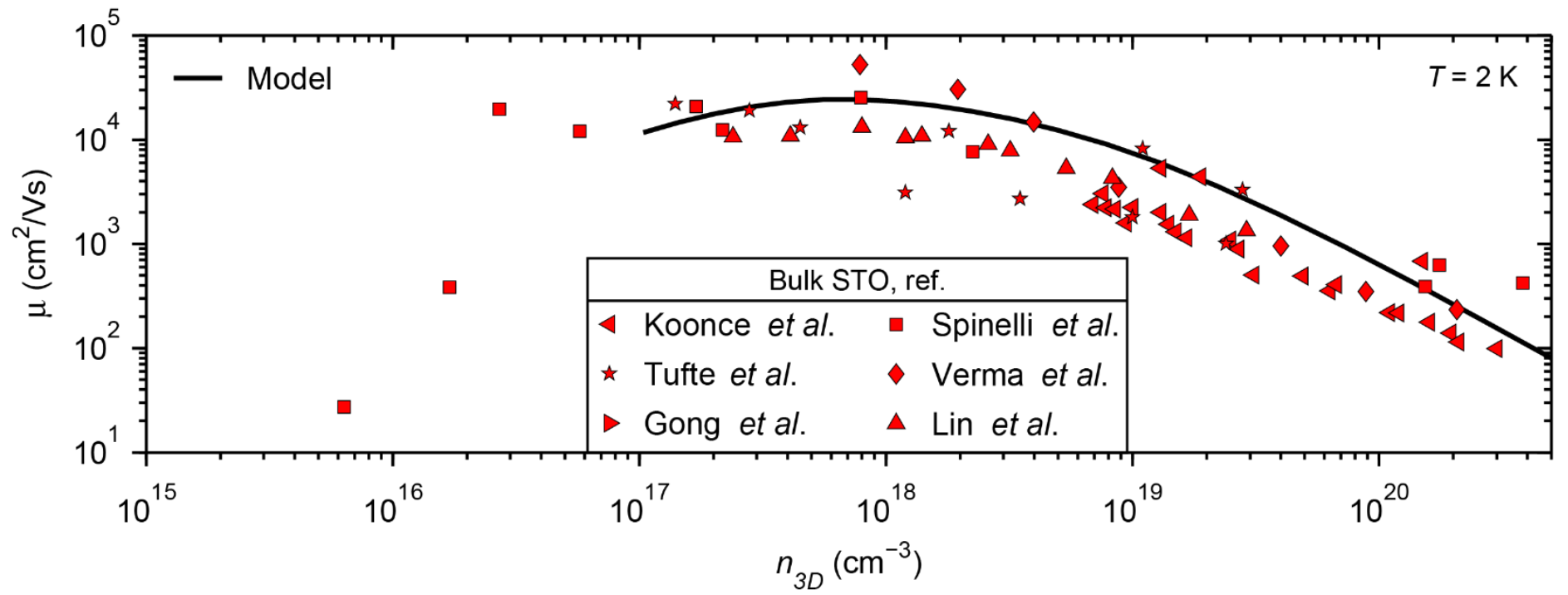

Figure 5 - The low-temperature electron mobility of bulk conducting STO change drastically as the charge carrier density is varied. The data is obtained from Tufte et al. [23], Koonce et al. [25], Verma et al. [18], Spinelli et al. [40], Gong et al. [41], Lin et al. [42]. The model is obtained by interpolating between the three different scattering regimes described in Section 3.3.

\section{3 - Electron mobility at low temperature}

Opposed to the situation at room temperature, the low-temperature electron mobility in STO is very sensitive to variations in the charge carrier density (see Figure 5).

As mentioned above, the low-temperature electron mobility is limited by scattering with ionized impurities. These charged impurities come in two varieties: ionized background impurities and ionized intentional impurities. In the case of the former, the possible background donors include oxygen vacancies, while common background acceptors include strontium vacancies, aluminium or iron [41-43]. In the case of the latter, the common intentional donors include oxygen vacancies, niobium and lanthanum. As these impurities ionize upon incorporation into the STO lattice due to donor-acceptor compensation, there reside substantial concentrations of scattering centres for charge carriers in STO. Usually, the concentration of background impurities is around $N_{s} \approx 5 \times 10^{18} \mathrm{~cm}^{-3}$ whereas the concentration of intentional impurities naturally depends on the doping

\section{4 - Electron mobility of strained STO}

Until this point, we have been dealing with the electron mobility of STO having an unperturbed and intrinsic lattice.

The effect of a strained STO lattice on electron mobility was studied by Jalan et al. [44]. Impressively, the authors found that the electron mobility of La-doped STO thin films under uniaxial stress could be substantially increased along the direction of the stress. For one of the two different carrier densities studied, the electron mobility exceeded a staggering $120,000 \mathrm{~cm}^{2} / \mathrm{Vs}$ at $1 \mathrm{~K}$ (see Figure 6). Importantly, the authors note that through strain engineering it could be possible to realise STO under biaxial stress with a uniform high electron mobility. It is interesting to observe that, in this study, the effect of stress was only affecting the lowtemperature electron mobility and for $T>20 \mathrm{~K}$ did not seem to change the transport properties noticeably. This may indicate that the strain effect is primarily affecting the scattering contribution from ionized impurities. 

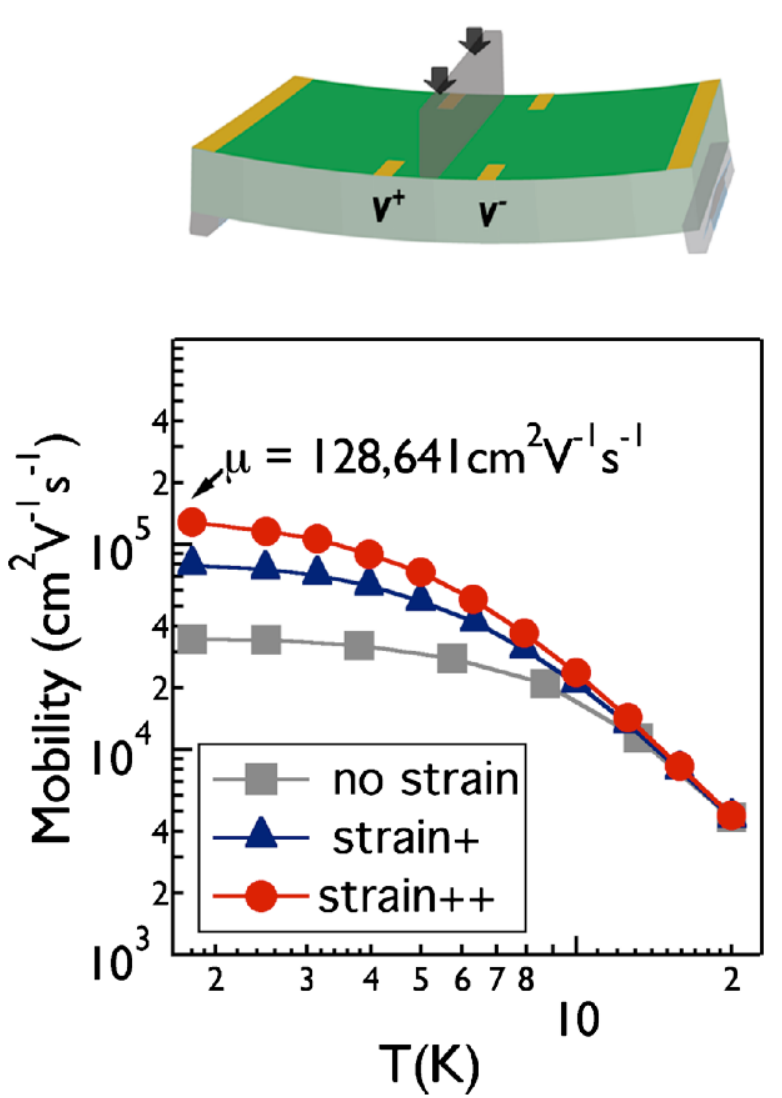

Figure 6 - By applying a uniaxial stress, the electron mobility of STO can be increased significantly. Reprinted from reference [44], with the permission of AIP Publishing.

Conversely, Kobayashi et al. studied the effect of deliberately introducing strontium vacancy clusters in Nb-doped STO [45]. Due to the strontium vacancy clusters, the STO films were remarkably shown to exhibit enhanced electron mobilities exceeding $50,000 \mathrm{~cm}^{2} / \mathrm{Vs}$ at $2 \mathrm{~K}$ (see Figure 7). According to the authors, this behaviour was due to the strontium vacancy clusters creating a compressive strain field throughout the STO crystal. Opposed to the above case of uniaxial strained STO, this strain field manifested itself in an increase of the electron mobility for the entire temperature range studied $(T=2-300 \mathrm{~K})$. Therefore, it is apparent that these strontium vacancy clusters may affect, not only a single but possibly, several scattering mechanisms.

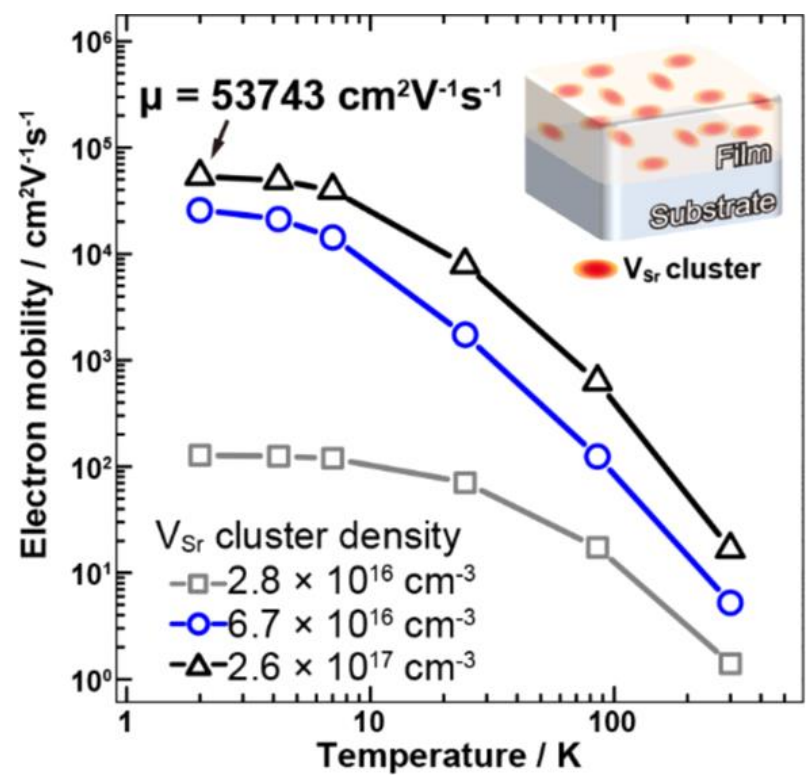

Figure 7 - Straining the STO locally, through defect engineering, can markedly increase the electron mobility in the entire temperature range from $T=2-300 \mathrm{~K}$. Reprinted with permission from reference [45]. Copyright 2015 American Chemical Society.

In order to understand the effect of strain in STO on electron mobility, Janotti et al. modelled biaxially strained STO using first-principles calculations [46]. Here, they found that STO with tensile biaxial stress in the (100) or (110) planes indeed had a lowered effective mass of the lowest energy conduction band along the transport directions.

From the above studies, it is clear that strain is an effective pathway to increase the low-temperature electron mobility, and in some cases even increase it above the intrinsic upper limit dictated by ionized background impurities of $\sim 30.000 \mathrm{~cm}^{2} / \mathrm{Vs}$ at $2 \mathrm{~K}$. The usual way to design and control the strain of a given material is to place it in proximity with another material in a so-called heterostructure. As this has been studied extensively in the case of STO, the electron mobility of STO-based heterostructures will be the topic of the following Section 4 .

\section{Section 4: Electron mobility in confined STO-based systems}

Besides the strain-approach described in the previous Section, the electron mobility has been enhanced beyond what is usually observed in bulk conducting STO by confining the electrons to a thin sheet. This is achieved by breaking the lattice symmetry either by doping only a confined region with, e.g., La donors ( $\delta$-doping) or by forming a heterostructure where STO is interfaced with another material. In both these cases, the electrons are confined to reside near the dopant layer or interface. A careful control of the local scattering landscape underpins the success of modulation doping where the donors are moved to a layer spatially separated from the electrons. The downside of this is, however, that the lattice symmetry breaking may introduce additional scattering mechanisms such as interface roughness scattering as described in Section 2.4.

Specifically, confined conductivity may be formed by doping STO locally either by: i) intentional dopants such as La, ii) electronic reconstructions due to polar discontinuities across the heterostructure or iii) introduction of oxygen vacancies. In STO-based heterostructures, the translation symmetry near the interface is broken and the previously degenerate $t_{2 g}$ levels split further in accordance with their specific orbital orientation. 
These split $t_{2 g}$ levels are comprised of the $3 d_{x y}, 3 d_{x z}$ and $3 d_{y z}$ levels, which usually all are populated in confined STO-based systems [26].

In particular, the confined conductivity - i.e. a two-dimensional electron (2DEG) gas - found at the $\mathrm{LaAlO}_{3} / \mathrm{SrTiO}_{3}$ interface has been studied extensively for the past fourteen years. However, its $p$-type counterpart the two-dimensional hole gas (2DHG) - has proved elusive. Very recently it was demonstrated that a highly mobile $2 \mathrm{DHG}$ is realisable in epitaxially grown $\mathrm{SrTiO}_{3} / \mathrm{LaAlO}_{3} / \mathrm{SrTiO}_{3}$ heterostructures $[47,48]$. This provides a platform for future applications using confined electron-hole systems.

In the following, we will review a representative selection of different highmobility STO-based material systems with confined electrons, and outline the mechanisms proposed to explain the mobility enhancement.

\section{1 - Temperature dependence of the mobility}

The transport properties of a range of high-mobility STO-based material systems are summarized in Figure 8. For the majority of the systems, it is evident from Figure 8 that the carrier densities are temperature independent. At low temperatures, the mobility varies greatly from one material system to another with $\mathrm{y}-\mathrm{Al}_{2} \mathrm{O}_{3} / \mathrm{SrTiO}_{3}$ exhibiting the highest mobility exceeding $100,000 \mathrm{~cm}^{2} / \mathrm{Vs}$ at $2 \mathrm{~K}$. Several other heterostructures also obtains high electron mobilities larger than $20,000 \mathrm{~cm}^{2} / \mathrm{Vs}$ at $2 \mathrm{~K}$. Similar to bulk conducting STO, the mobility at the intermediate temperatures roughly varies as $\sim T^{-2}$ characteristic for electron-electron scattering up to $T \sim 150 \mathrm{~K}$ where longitudinal optical phonon scattering often becomes dominant. The effect of this electron-electron scattering contribution is similar to that in Figure 2.

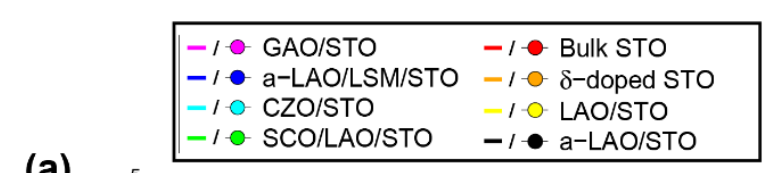

(a)
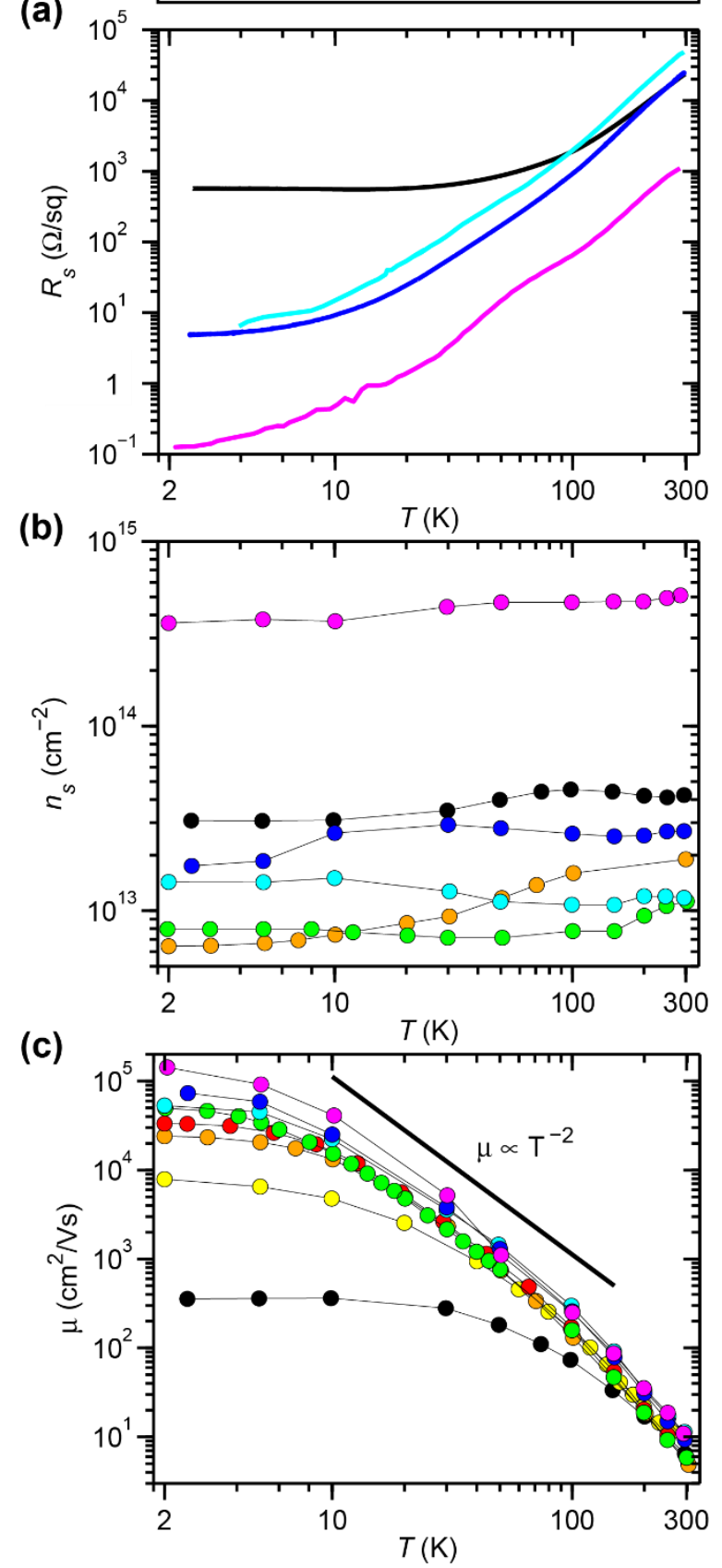

Figure 8 - The sheet resistance, carrier density and electron mobility of a range of different confined STO-based systems along with bulk conducting STO. Note that the data presented here represent the highest reported electron mobilities of the respective systems. The data is obtained from: $\mathrm{Al}_{2} \mathrm{O}_{3} / \mathrm{SrTiO}_{3}$ (GAO/STO) [6]; amorphous-LaAlO ${ }_{3} / \mathrm{LaSrMnO}_{3} / \mathrm{SrTiO}_{3}$ (aLAO/LSM/STO) [49]; $\mathrm{CaZrO}_{3} / \mathrm{SrTiO}_{3}$ (CZO/STO) [50]; $\mathrm{SrCuO}_{2} / \mathrm{LaAlO}_{3} / \mathrm{SrTiO}_{3}$ (SCO/LAO/STO) [51]; bulk $\mathrm{SrTiO}_{3}$ (STO) [52]; $\delta$-doped $\mathrm{SrTiO}_{3}$ ( $\delta$-doped STO) [53]; $\mathrm{LaAlO}_{3} / \mathrm{SrTiO}_{3}$ (LAO/STO) [54]; amorphous-LaAlO ${ }_{3} / \mathrm{SrTiO}_{3}$ (aLAO/STO) [55]. Common for these high-mobility systems are that the carrier density often only is little temperature dependant, whereas the electron mobility can differ by up to four orders of magnitude from room temperature to $2 \mathrm{~K}$. At intermediate temperatures between $T=10-150$ $\mathrm{K}$, the electron mobility follows an overall $\mu \propto \sim T^{-2}$ dependence characteristic for electron-electron scattering. 


\section{2 - Mobility at room temperature}

For both bulk conducting STO and two-dimensionally confined electron gases in STO, the room temperature mobility is generally less than 12 $\mathrm{cm}^{2} / \mathrm{Vs}$ (see Figure 3 and Figure 8 ). The room temperature mobility is limited by longitudinal optical phonon scattering or electron-electron scattering. Moderate variations ranging from 2 to $12 \mathrm{~cm} / \mathrm{Vs}$ is observed upon varying the growth conditions, confinement, carrier density and material systems. There is a tendency for obtaining a larger room temperature mobility when increasing the carrier density as for example observed in $\mathrm{y}-\mathrm{Al}_{2} \mathrm{O}_{3} / \mathrm{SrTiO}_{3}$.

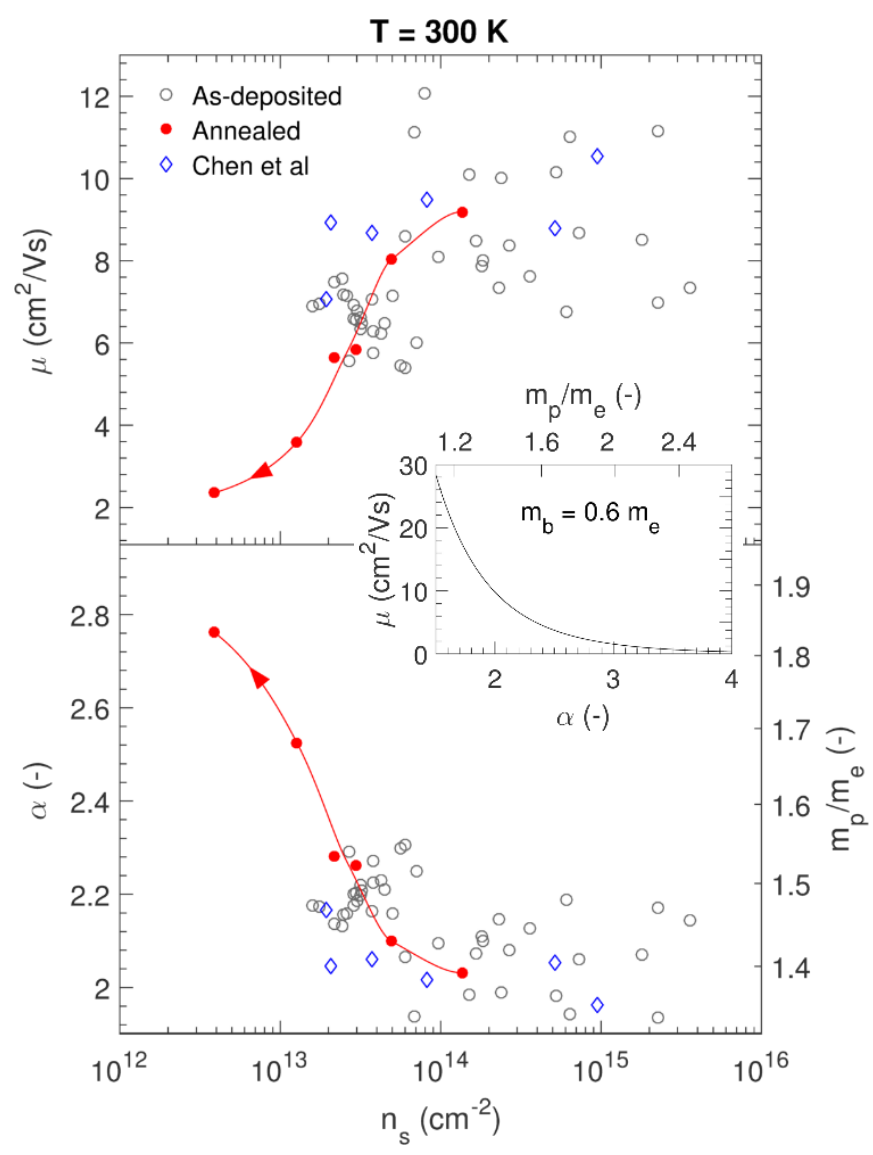

Figure 9 - Electron mobility $(\mu)$, electron-phonon coupling $(\alpha)$ and polaron mass $\left(m_{p} / m_{e}\right)$ as a function of the Hall carrier density $\left(n_{s}\right)$ for $\gamma$ $\mathrm{Al}_{2} \mathrm{O}_{3} / \mathrm{SrTiO}_{3}$ heterostructures with varying carrier densities obtained by varying the deposition conditions or performing post-annealing in 1 bar oxygen at $200{ }^{\circ} \mathrm{C}$. Reprinted figure with permission from [56]. Copyright 2018 by the American Physical Society.

\subsection{1 - $-\mathrm{Al}_{2} \mathrm{O}_{3} / \mathrm{SrTiO}_{3}$}

The interface between spinel $\gamma-\mathrm{Al}_{2} \mathrm{O}_{3}$ and perovskite STO becomes $n$-type conducting as oxygen vacancies are formed in the near-interface region of STO $[6,57]$. The sheet carrier density $\left(n_{s}\right)$ can be tuned by varying the deposition conditions or performing a post-annealing step in an oxygencontaining atmosphere after the deposition [57]. The room temperature mobility was found to be enhanced as the carrier density increased, reaching a value up to $12 \mathrm{~cm}^{2} / \mathrm{Vs}$ at $n_{s} \geq 8 \times 10^{13} \mathrm{~cm}^{-2}$ (see Figure 9). When decreasing the carrier density, the mobility reduces to $2 \mathrm{~cm}^{2} / \mathrm{Vs}$ at $n_{s}=4 \times 10^{12} \mathrm{~cm}^{-2}$. Curiously, the same trend is observed for bulk conducting STO (see Figure 3).

Similar to the case in bulk STO, the limitations of the room temperature mobility in $\mathrm{\gamma}-\mathrm{Al}_{2} \mathrm{O}_{3} / \mathrm{SrTiO}_{3}$ was attributed by Christensen et al. to interaction with longitudinal optical phonons, which scatter both the electrons and increases the effective mass by polaron formation [56]. Using the expression for the phonon scattering (see Section 2.2 and Equations 8$10)$, the electron-phonon coupling $(\alpha)$ and the polaron effective mass enhancement $\left(m_{p} / m_{e}\right)$ was calculated from the mobility as presented in Figure 9. From this, it was deduced that the mobility enhancement at high carrier densities is caused by electron screening which weakens the electron-phonon coupling and lowers the effective polaron mass.

\section{3 - Mobility at low temperature}

Analogous to bulk conducting STO, the low-temperature electron mobility of confined STO-based systems is likewise strongly carrier density dependent. The subsequent subsection will introduce the different confined STO-based systems, previously studied at low temperature, and provide an overview of this dependence.

\subsection{1 - $\delta$-doped STO}

Through state-of-the-art deposition techniques, it is possible to meticulously engineer the dopant profile in oxides such as STO. This degree of lattice engineering has provided the possibility to realise so-called $\delta$ doped STO where all charge carrier dopants are embedded in a layer with thickness less than $10 \mathrm{~nm}$ between two slabs of insulating and nominally un-doped STO. This confinement of the electrons, result in a symmetric quantum well with essentially the same physical properties as observed for electrons confined at oxide heterointerfaces. More importantly, this method, perfected for semiconductors and simple oxides such as ZnO (see Section 5), is a commonly used pathway to augment the electron mobility of a given system. $\delta$-doped STO has been realised experimentally by use of pulsed laser deposition (PLD) [58] and molecular beam epitaxy (MBE) $[53,59]$. In these previous studies, the dopant used in PLD and MBE growth of $\delta$-doped STO were niobium [58] and lanthanum [59],[53], respectively. Figure 10 shows the available data for electron mobility of $\delta$-doped STO at $T=2 \mathrm{~K}$ realised by PLD and MBE. It is evident from the figure that the highest observed electron mobilities of $\mu=20.000 \mathrm{~cm}^{2} / \mathrm{Vs}$ coincide with the lowest carrier densities at $n_{s}=\sim 10^{12} \mathrm{~cm}^{-2}$. This behaviour seems at first glance to qualitatively agree with what is the case for bulk conducting STO (see Figure 5). However, the electron mobility at larger carrier densities where $n_{s}>10^{14} \mathrm{~cm}^{-2}$ seem not to reduce as rapidly as is the case for bulk STO but rather saturate around a mobility of $\mu=1000 \mathrm{~cm}^{2} / \mathrm{Vs}$. The origin of the high electron mobility in $\delta$-doped STO was in all previous studies attributed to the absence of background impurities in the $\delta$-doped STO layer due to the purity of the growth sources $[53,58,59]$. 

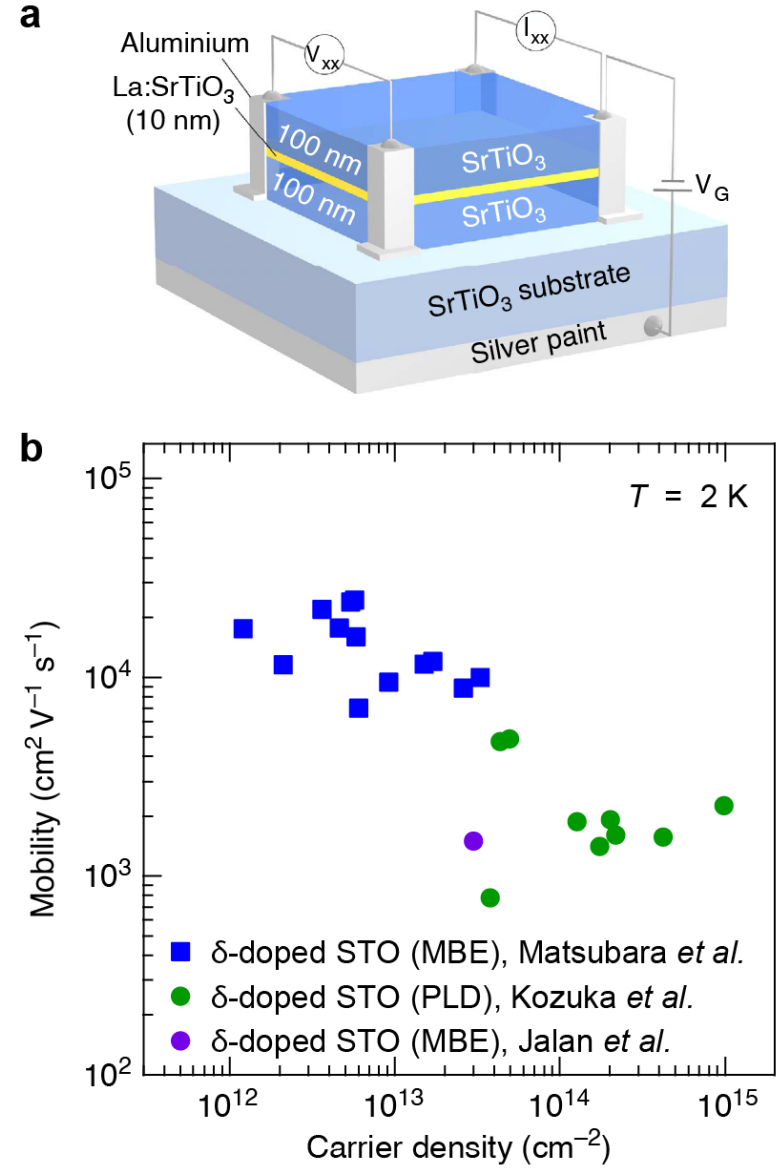

Figure 10 - The low-temperature electron mobility of $\delta$-doped STO is gradually decreased as the carrier density is increased. This behaviour qualitatively resembles the trend in bulk conducting STO. The figure is adapted from reference [53] and the data is obtained from Kozuka et al. [58], Jalan et al. [59], Matsubara et al. [53].

\subsection{2 - $\mathrm{LaAlO}_{3} / \mathrm{SrTiO}_{3}$}

The active field of confined electron gases in STO-based heterostructures was initiated in 2004 when it was found by Ohtomo \& Hwang that the interface between the insulators LAO and STO could be made conducting [5]. Moreover, when the LAO film was deposited at an oxygen partial pressure of approximately $10^{-6} \mathrm{mbar}$, an electron mobility in excess of $10,000 \mathrm{~cm}^{2} / \mathrm{Vs}$ could be realized. This enabled the observation of clear Shubnikov-de Haas quantum oscillations of the resistance as a function of the magnetic field. The oscillations were observed both when the magnetic field was applied perpendicular and parallel to the interface. This suggested that the highly mobile electrons obtained by growth at this low oxygen partial pressure were not confined to a narrow sheet at the interface. The three-dimensional conductivity was in later studies by Herranz et al. and Kalabukhov et al. confirmed to be due to oxygen vacancies homogeneously distributed in the bulk of STO formed during the deposition of LAO $[60,61]$. This is shown in Figure 11 where the LAO/STO heterostructure grown at a low oxygen pressure exhibits a very high sheet electron density, a high electron mobility and a strong bluish cathode luminescence stemming from oxygen vacancies [61]. The confined nature of the electrons can be obtained either by post-annealing in oxygen or by growth at a higher oxygen partial pressure, which reduces the amount of oxygen vacancies in the bulk of STO. It is still debated whether these confined electrons stem from, e.g., residual oxygen vacancies in STO or oxygen vacancies spontaneously formed on the LAO surface to alleviate the polar catastrophe [62]. Irrespectively of the origin of the electrons, confining the electrons at the LAO/STO interface is generally observed to result in electron mobility values on the order of $1,000 \mathrm{~cm}^{2} / \mathrm{Vs}$.

The mobility of interface-confined electrons in LAO/STO has been enhanced through optimization of the deposition parameters [10,54]. Here, a decrease in the growth pressure from $900{ }^{\circ} \mathrm{C}$ to $650{ }^{\circ} \mathrm{C}$ resulted in an increase of the electron mobility from 600 to $8,000 \mathrm{~cm}^{2} / \mathrm{Vs}$ (see Figure 12) along with an emergence of Shubnikov-de Haas oscillations only appearing with magnetic fields applied perpendicular to the interface. The mobility enhancement was found to occur together with an order of magnitude decrease in the total carrier density reaching a value of $5 \times 10^{12}$ $\mathrm{cm}^{-2}$. Interestingly, the observation of high electron mobilities coinciding with low carrier densities appears to be a general trend for ungated LAO/STO [63-65] as shown in Figure 13. The same figure also shows that surface treatments using conducting atomic force microscopy, solvent deposition and heating can be used to enlarge the mobility up to 20,000 $\mathrm{cm}^{2} / \mathrm{Vs}$ at a low carrier density of $1.5 \times 10^{12} \mathrm{~cm}^{-2}$.

Several other pathways for enhancing the electron mobility in LAO/STO have been reported in the literature, including electrostatic gating $[66,67]$ as well as inserting a $\mathrm{La}_{1-\mathrm{x}} \mathrm{Sr}_{x} \mathrm{MnO}_{3}$ buffer layer [49] between LAO and STO or a $\mathrm{SrCuO}_{2}$ capping layer [51] on top of LAO/STO during growth. The latter two pathways will be discussed further in the next two subsections.

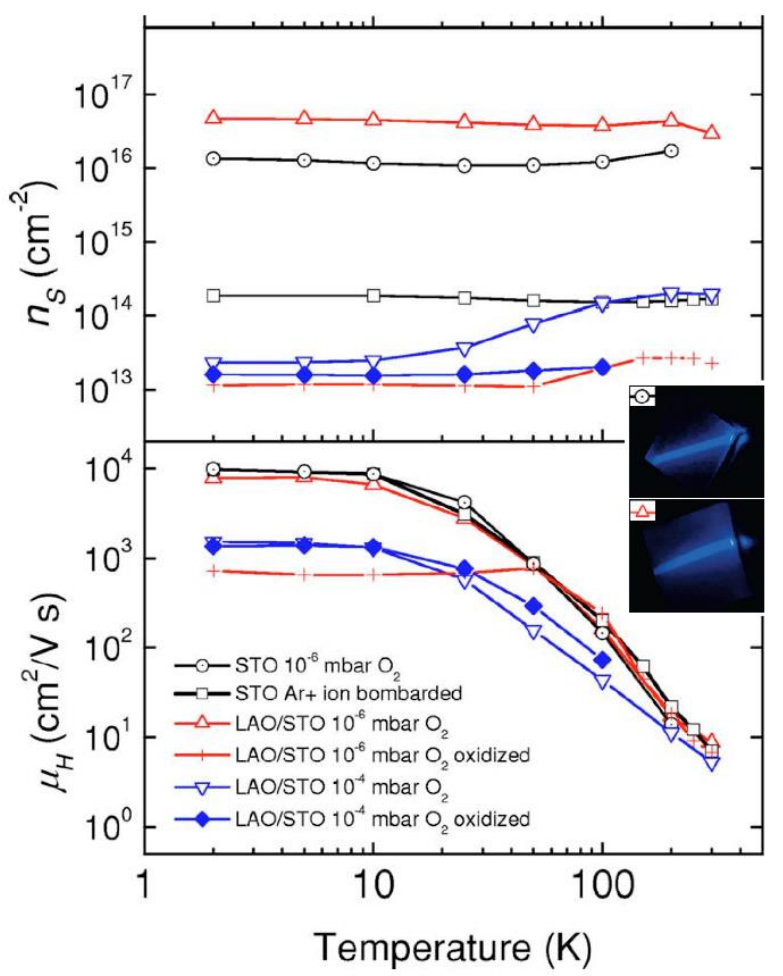

Figure 11 - The Hall carrier density $\left(n_{S}\right)$ and mobility $\left(\mu_{H}\right)$ as a function of temperature for (i) STO vacuum annealed at $800^{\circ} \mathrm{C}$ in an oxygen pressure of $10^{-6} \mathrm{mbar}$, (ii) STO exposed to argon ion bombardment with an energy of $300 \mathrm{eV}$ and a current density of $0.2 \mathrm{~mA} / \mathrm{cm}^{2}$ and (iii) LAO/STO deposited at $800^{\circ} \mathrm{C}$ in an oxygen pressure of $10^{-4}$ or $10^{-6} \mathrm{mbar}$ with or without a twohour postannealing step at $600{ }^{\circ} \mathrm{C}$ in 500 mbar oxygen pressure during cool-down after the deposition. Reprinted figure with permission from [61]. Copyright 2007 by the American Physical Society. 


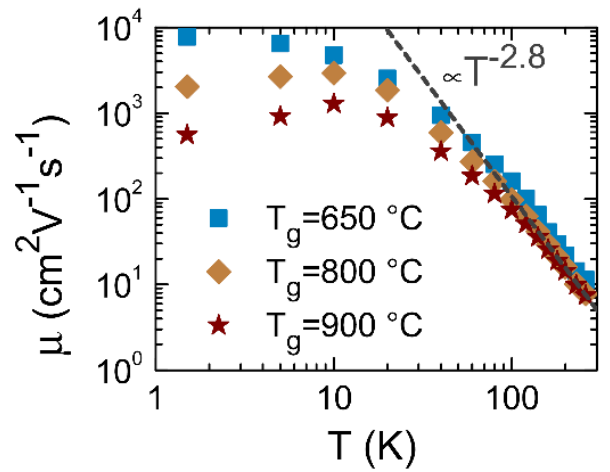

Figure 12 - Electron mobility $(\mu)$ as a function of temperature $(T)$ for three LAO/STO heterostructures where LAO was deposited at growth temperatures ranging from $T_{g}=650$ to $900{ }^{\circ} \mathrm{C}$. Reprinted from reference [54], with the permission of AIP Publishing.

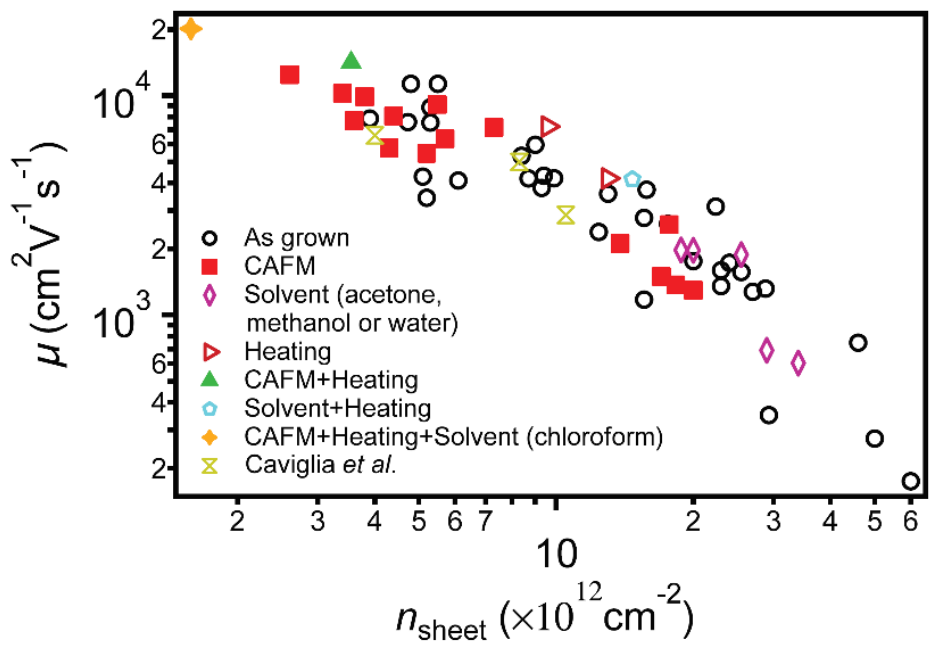

Figure 13 - The electron mobility $(\mu)$ as a function of the sheet carrier density $\left(n_{\text {sheet }}\right)$ compiled for a range of LAO/STO heterostructures with or without treating the surface with conducting atomic force microscopy (CAFM), addition of various solvents and/or heating. All data were acquired at $2 \mathrm{~K}$ or less. The figure is adapted from reference [64], which includes data from Caviglia et al. [10].

\subsection{3 - Amorphous- $-\mathrm{LaAlO}_{3} / \mathrm{LaSrMnO}_{3} / \mathrm{SrTiO}_{3}$}

One pathway to increase the electron mobility beyond what typically is observed in LAO/STO of around $\sim 1,000 \mathrm{~cm}^{2} / \mathrm{Vs}$ is to insert a buffer layer of $\mathrm{La}_{1-x} \mathrm{Sr}_{x} \mathrm{MnO}_{3}$ between LAO and STO [49]. Inserting a $\mathrm{La}_{1-x} \mathrm{Sr}_{x} \mathrm{MnO}_{3}$ layer with a thickness of two unit cells or more leads to insulating interfaces, however, inserting a single unit cell reduces the carrier density and increases the electron mobility up to $70,000 \mathrm{~cm}^{2} / \mathrm{Vs}$ (see Figure 14). The reduction in the electron density were by Chen et al. attributed to occur by the $\mathrm{La}_{1-x} \mathrm{Sr}_{x} \mathrm{MnO}_{3}$ layer acting as an electron sink thus localizing part of the electrons leaving the residual electrons to populate the conduction band in STO (see Figure 14). The low oxidation potential of $\mathrm{La}_{1-x} \mathrm{Sr}_{x} \mathrm{MnO}_{3}$ was thought by the authors to minimize the oxygen vacancy formation in STO and hence reduce the ionized donor scattering. As the oxygen vacancy concentration in STO were lowered, the conducting electrons were suggested to originate from the LAO layer. This was expected to result in a separation of donors and charge carriers in accordance with the modulation doping strategy, leading to the observed mobility enhancement.
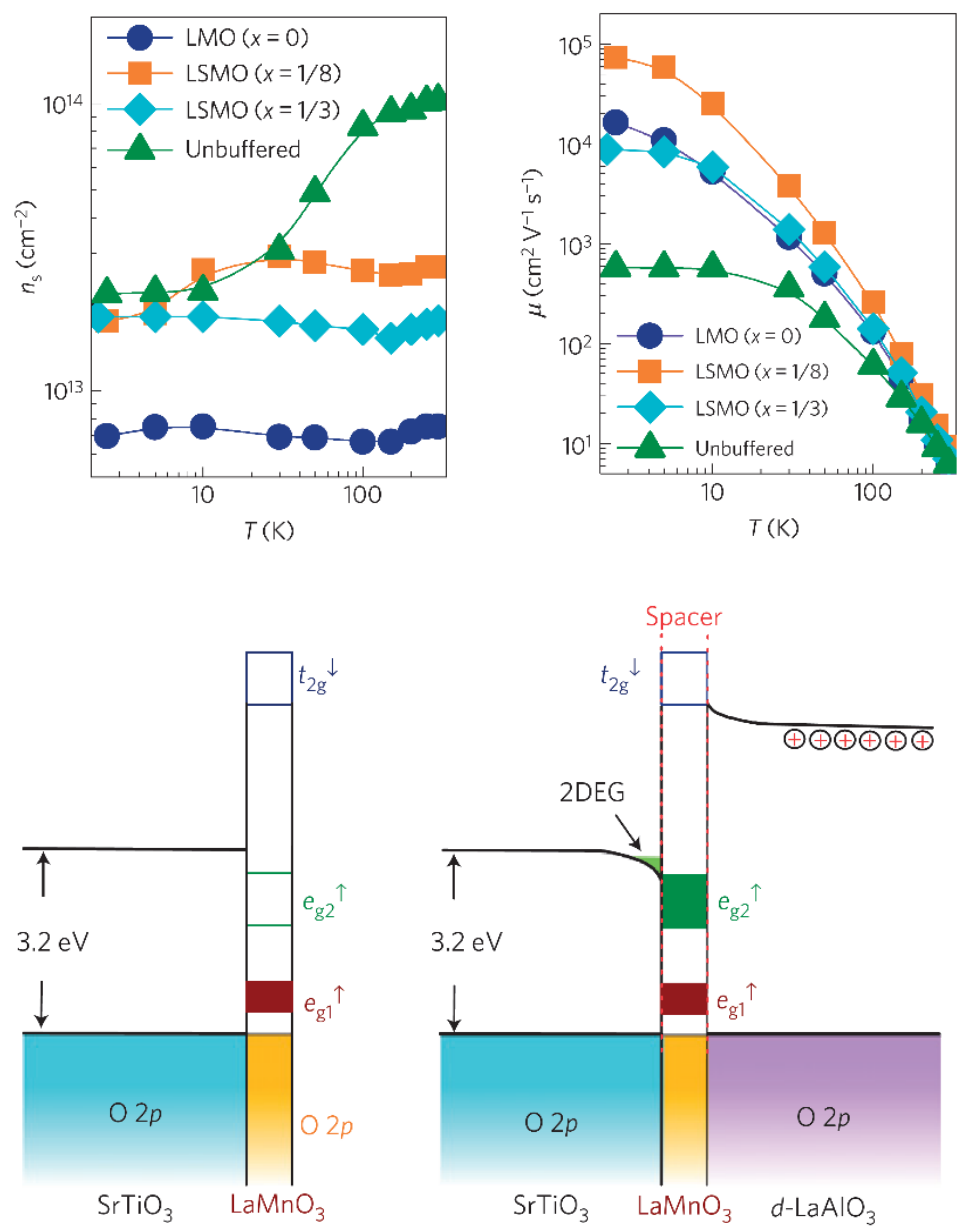

Figure 14 - The carrier density $\left(n_{s}\right)$ and mobility $(\mu)$ as a function of temperature for room-temperature grown amorphous-LAO (d-LaAlO ${ }_{3}$ ) deposited directly on STO (unbuffered) or on $\mathrm{La}_{1-\mathrm{x}} \mathrm{Sr}_{\mathrm{x}} \mathrm{MnO}_{3}$ (1uc)/SrTiO with $x=0,1 / 8$ or $1 / 3$. The lower part of the figure shows the band alignment of the bands in $\mathrm{STO}, \mathrm{LaMnO}_{3}$ and d- $\mathrm{LaAlO}_{3}$ showing that the electrons first occupies the $\mathrm{LaMnO}_{3}$ layer before forming a conducting electron gas at the near-interface region of STO. Reprinted by permission from the RightsLink Permissions Springer Customer Service Centre GmbH: Springer Nature, Nature Materials, [49], 2015. 


\subsection{4 - $\mathrm{SrCuO}_{2} / \mathrm{LaAlO}_{3} / \mathrm{SrTiO}_{3}$}

It was demonstrated by Huijben et al. that depositing STO with additional top layers after the normal growth of LAO could enhance the electron mobility [51]. Specifically, when a usually grown LAO/STO heterostructure was capped with one unit cell of $\mathrm{SrCuO}_{2}$ and an additional two unit cells of STO, the resulting conducting LAO/STO interface was shown to display enlarged electron mobilities exceeding $50,000 \mathrm{~cm}^{2} / \mathrm{V}$ at $2 \mathrm{~K}$ (see Figure 15). The authors attributed this large enhancement in electron mobility to a reduction in the amount of oxygen vacancies at the LAO/STO interface that in turn would correspond to a reduced ionized impurity scattering of electrons [51]. The highest observed electron mobility exceeding 50,000 $\mathrm{cm}^{2} / \mathrm{V}$ at $2 \mathrm{~K}$ was realised when growing the $\mathrm{SrCuO}_{2}$ capped LAO/STO heterostructure at the lowest studied oxygen partial pressure of $10^{-6} \mathrm{mbar}$.

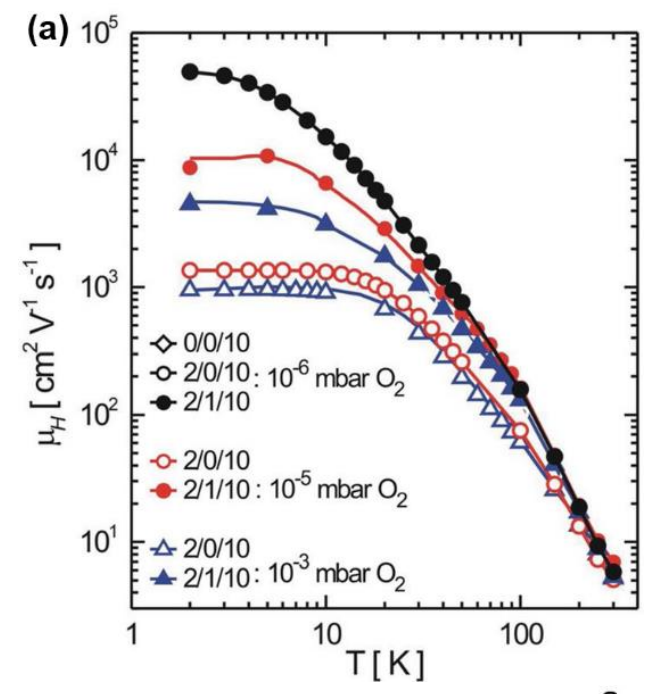

(b)
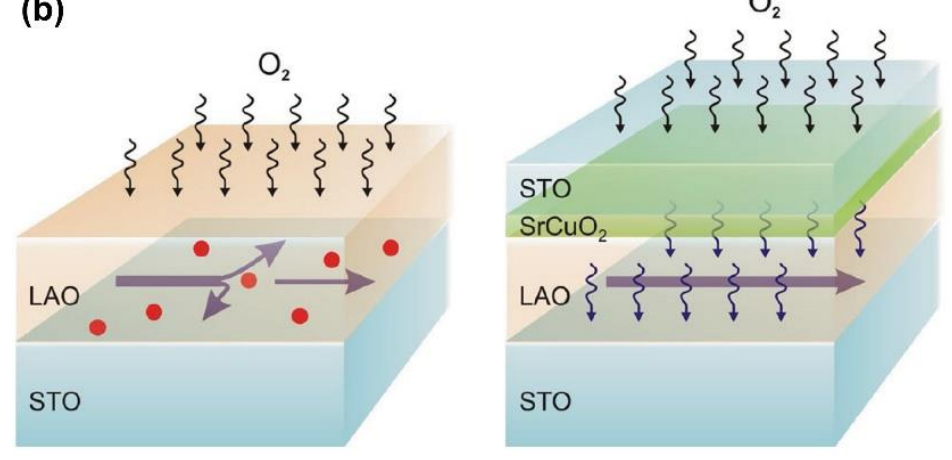

Figure 15 - (a) The electron mobility, $\mu_{H}$, as a function of temperature, $\mathrm{T}$, for $\mathrm{SrCuO}_{2}$ capped LAO/STO heterostructures (filled markers) and identically prepared structures without the $\mathrm{SrCuO}_{2}$ capping layer (open markers). Evidently, inclusion of the $\mathrm{SrCuO}_{2}$ capping layer results in greatly enlarged mobility values. The highest observed electron mobility was realised in the $\mathrm{SrTiO}_{3}\left(2\right.$ u.c.) $/ \mathrm{SrCuO}_{2}$ (1u.c.) $/ \mathrm{LaAlO}_{3}\left(10\right.$ u.c.) $/ \mathrm{SrTiO}_{3}$ heterostructure $(2 / 1 / 10)$ grown at $10^{-6} \mathrm{mbar}$. (b) The proposed mechanism for the enhanced interface electron mobility, where the $\mathrm{SrCuO}_{2}$ is thought to prevent the formation of interfacial LAO/STO oxygen vacancies and thereby reduce electron ionized impurity scattering. The figure is adapted from reference [51].

\subsection{5 $-\gamma-\mathrm{Al}_{2} \mathrm{O}_{3} / \mathrm{SrTiO}_{3}$}

The heterostructure composed of spinel $\mathrm{\gamma}-\mathrm{Al}_{2} \mathrm{O}_{3}$ epitaxially grown on perovskite STO exhibits the, to date, largest electron mobility of 140,000 $\mathrm{cm}^{2} / \mathrm{Vs}$ at $2 \mathrm{~K}$ observed in any STO-based electronic system [6]. The high electron mobility was observed to peak at a sheet carrier density of around $4 \times 10^{14} \mathrm{~cm}^{-2}$ (see Figure 16) in contrast to LAO/STO where the highest mobilities are observed at much lower carrier densities of around $\sim 10^{12}$ $\mathrm{cm}^{-2}$. Interestingly, it was shown by Christensen et al. that a gentle ex-situ annealing at $20-100{ }^{\circ} \mathrm{C}$ following growth enhanced the mobility without affecting the carrier density [68]. On the contrary, a harsher annealing at $200{ }^{\circ} \mathrm{C}$ was shown to lead to a decrease in both carrier density and electron mobility [56]. Photoemission studies and density functional theory calculations allowed the authors to suggest that the oxygen vacancy donors are more stable at the $\mathrm{y}-\mathrm{Al}_{2} \mathrm{O}_{3} / \mathrm{SrTiO}_{3}$ interface [68]. Based on this, it was proposed that the high mobility is caused by a lowering of the electron-donor scattering from having oxygen vacancies preferentially located at the interface with the electrons extending deeper into STO (see Figure 16).

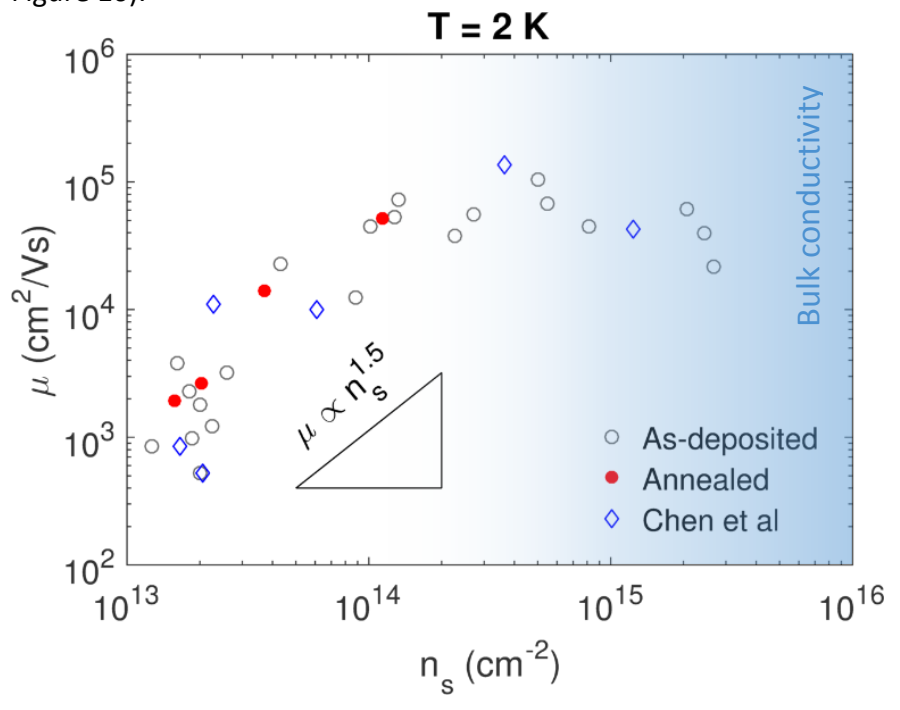

High- $\mu \gamma-\mathrm{Al}_{2} \mathrm{O}_{3} / \mathrm{SrTiO}_{3}$

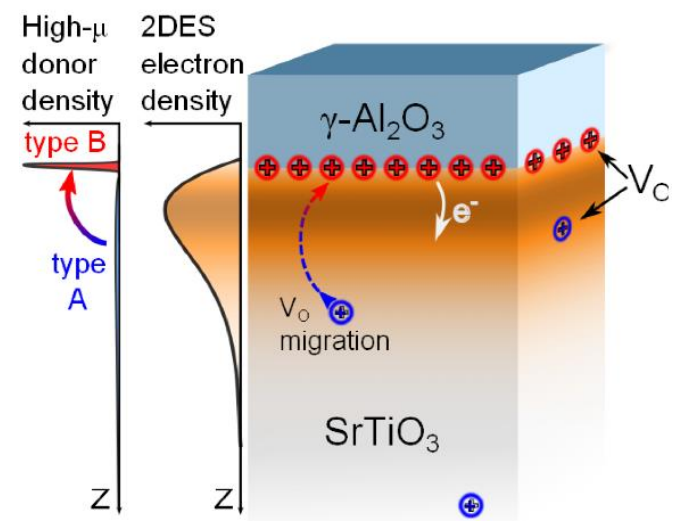

Figure 16 - (a) Electron mobility $(\mu)$ as a function of carrier density $\left(n_{s}\right)$ for $\mathrm{V}-\mathrm{Al}_{2} \mathrm{O}_{3} / \mathrm{SrTiO}_{3}$ heterostructures with varying carrier densities obtained by varying the deposition conditions or performing post-annealing in 1 bar oxygen at $200{ }^{\circ} \mathrm{C}$. Reprinted figure with permission from [56]. Copyright 2018 by the American Physical Society. (b) Schematic illustration of the proposed mechanism for the high-mobility in which a lowering of the electron-donor scattering occurs as oxygen vacancies primarily are thought to locate at the interface whereas electrons extend deeper into STO. Reprinted figure with permission from [68]. Copyright 2017 by the American Physical Society. 


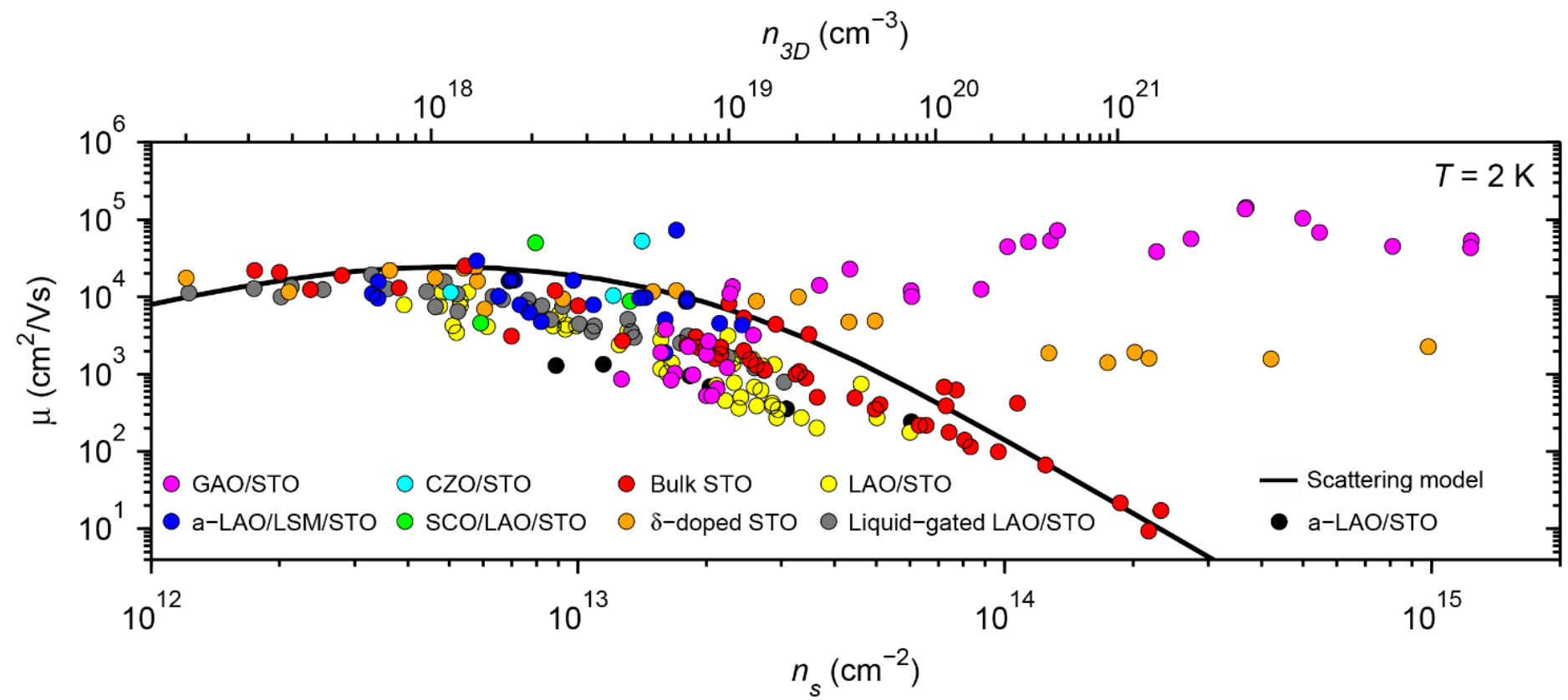

Figure 17 - The dependence of the low-temperature electron mobility, $\mu$, on the sheet carrier density, $n_{s}$, as well as corresponding bulk carrier density, $n_{3 D}$, for bulk conducting STO and confined STO-based systems. The scattering model is obtained by interpolating between the three different scattering regimes described in Section 3.3. The data is obtained from: $\gamma-\mathrm{Al}_{2} \mathrm{O}_{3} / \mathrm{SrTiO}_{3}$ (GAO/STO) [6]; amorphous-LaAlO $3 / \mathrm{LaSrMnO}_{3} / \mathrm{SrTiO}_{3}(\mathrm{a}-\mathrm{LAO} / \mathrm{LSM} / \mathrm{STO})$ [49];

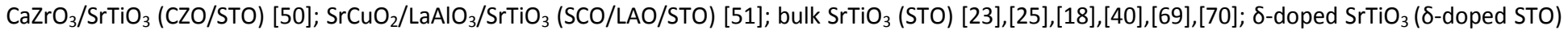
[53]; $\mathrm{LaAlO}_{3} / \mathrm{SrTiO}_{3}$ (LAO/STO) [63,64]; ion liquid-gated $\mathrm{LaAlO}_{3} / \mathrm{SrTiO}_{3}$ (liquid-gated LAO/STO) [67]; amorphous-LaAlO $3 / \mathrm{SrTiO}_{3}(\mathrm{a}-\mathrm{LAO} / \mathrm{STO}$ ) [55]

\subsection{6 - Overview of low-temperature electron mobility}

The previous Subsections 4.3.1-4.3.5 have provided a representative overview of the available literature for confined STO-based electronic systems with high mobility at low temperatures. Herein, the origin of enhanced electron mobility in the different systems was presented as suggested by the authors in the original papers. We have compiled the data into an overview of the low-temperature electron mobility as a function of carrier density for both bulk conducting STO and confined STO-based systems (Figure 17). The figure also includes the scattering model presented in Section 3.3. Note that in order to plot the electron mobility data for bulk conducting STO together with the confined electron systems, the three-dimensional carrier density was translated into the equivalent two-dimensional density following the expression derived by Trier et al. [65]. At first glance, it is clear from Figure 17 that some confined systems seem to follow an similar trend in $\mu\left(n_{s}\right)$ as bulk conducting STO over two orders of carrier densities for $10^{12} \mathrm{~cm}^{-2}<n_{s}<10^{14} \mathrm{~cm}^{-2}$. In particular, only $\delta$-doped STO and $\mathrm{\gamma}-\mathrm{Al}_{2} \mathrm{O}_{3} / \mathrm{SrTiO}_{3}$ with carrier densities larger than $n_{s}>\sim 10^{14} \mathrm{~cm}^{-2}$ falls markedly outside this overall electron mobility trend. At lower carrier densities, the observed electron mobilities of both these systems seem to merge with the trend of the other confined STO-based systems in addition to the trend of bulk STO. Although a few reported electron mobility values for STO-based systems at low carrier density significantly differs from the remaining data points, most electron mobility values seem to follow a common trend. In order to fully understand why the few outlying data points are falling outside this common trend, further investigations are needed to clarify the mechanism of their reduced scattering rates.

Since there, nonetheless, seem to exist a common behaviour of electron mobility between most confined systems and bulk STO, it points towards that the same scattering mechanisms in bulk STO are governing lowtemperature transport in confined STO-systems. In the low carrier density regime of $n_{s} \sim 10^{12}$, this suggest that the electron mobility in confined STOsystems overall is limited by scattering with ionized background impurities, i.e. the inherent purity and crystal quality of STO. In the large carrier density regime of $n_{s}>10^{13}$ the electron mobility reduction seems consistent with increased ionized donor scattering. In bulk STO these additional ionic scattering centres are formed by doping STO with e.g. oxygen vacancies or lanthanum. Therefore, the reduction of electron mobility observed for the confined STO-based systems having $n_{s} \sim 10^{13}$. $10^{14}$, with the exception of $\delta$-doped STO and $\mathrm{\gamma}-\mathrm{Al}_{2} \mathrm{O}_{3} / \mathrm{SrTiO}_{3}$, may be consistent with the dominant scattering mechanism being due to ionized donors. For some confined STO-based systems, this behaviour seems in agreement with how the authors in the original papers attributed the doping mechanism. In particular, confined STO-based systems where charge carriers are expected to originate from oxygen vacancies in STO is in good agreement with their trend in electron mobility. In these cases, control of the location and amount of the oxygen vacancy donors are generally believed to be essential for obtaining a high mobility. Conversely, charge carriers in LAO/STO are often attributed to arise due to the polarity discontinuity between LAO and STO within the so-called polar catastrophe model $[62,69]$. Although this model is expected to provide electrons to the LAO/STO interface, these electrons are not hypothesised to lead to ionized donors located in STO, but rather from the LAO surface similar to modulation doping. Therefore, the electron mobility trend of LAO/STO observed in Figure 17 therefore brings forth an interesting perspective on the polar catastrophe model as discussed by Trier et al. [65].

Concerning $\delta$-doped STO and $\gamma-\mathrm{Al}_{2} \mathrm{O}_{3} / \mathrm{SrTiO}_{3}$, there seem to be other origins for the scattering mechanism once the carrier density becomes large with $n_{s}>\sim 10^{14}$. However as presented in Subsection 4.3.5, recent transport, photo-electron spectroscopy and density functional studies of $\mathrm{V}-\mathrm{Al}_{2} \mathrm{O}_{3} / \mathrm{SrTiO}_{3}$ have shed some light on the ionized donor distribution at this particular interface [68]. Specifically, the authors came to the conclusion that oxygen vacancies in $\mathrm{\gamma}-\mathrm{Al}_{2} \mathrm{O}_{3} / \mathrm{SrTiO}_{3}$ were highly concentrated in STO right at the interface [68]. Whereas for $\delta$-doped STO there are less concrete suggestions in the literature why the electron mobility does not follow the remaining confined STO-based systems having $n_{s}>\sim 10^{14}$ and should be the subject of further investigations. 


\section{4 - One-dimensional confined conductivity}

The previous subsections 4.1-4.3 reviewed the literature concerning electron mobility for two-dimensionally confined STO-based heterointerfaces. Confining electrons in one dimension rather than the two-dimensional interfacial area seems to have a profound impact on the measured electron mobility as studied by Irvin et al. [70] In that study, LAO was deposited on STO at two different deposition conditions using a LAO thickness below the critical thickness for obtaining metallic conductivity. When a positively charged c-AFM tip was scanned at the surface of the insulating LAO/STO heterostructure, conductivity was formed locally underneath the tip (see Figure 18). In this way, the authors were able to write conducting lines and devices with nanoscale dimensions in a highly customizable way. When conducting squares with a side length ranging from $20 \mathrm{~nm}$ to $1 \mu \mathrm{m}$ were measured in the van der Pauw (vdP) geometry, the room temperature mobility was found to be on the order of $6-7 \mathrm{~cm}^{2} / \mathrm{Vs}$ (see Figure 18). This is consistent with the mobilities of unconfined and two-dimensional confined STO-based systems as discussed in the previous subsections 3.2 and 4.2. However, when conductivity was formed in the shape of 1.2-8 $\mu \mathrm{m}$ long Hall-bar, the mobility was found to increase significantly for Hall bar devices with widths below $100 \mathrm{~nm}$. The highest mobility of $350 \mathrm{~cm}^{2} / \mathrm{Vs}$ was obtained when the Hall bar width was $10 \mathrm{~nm}$. This mobility is astoundingly 60 times larger than what typically is observed in STO.
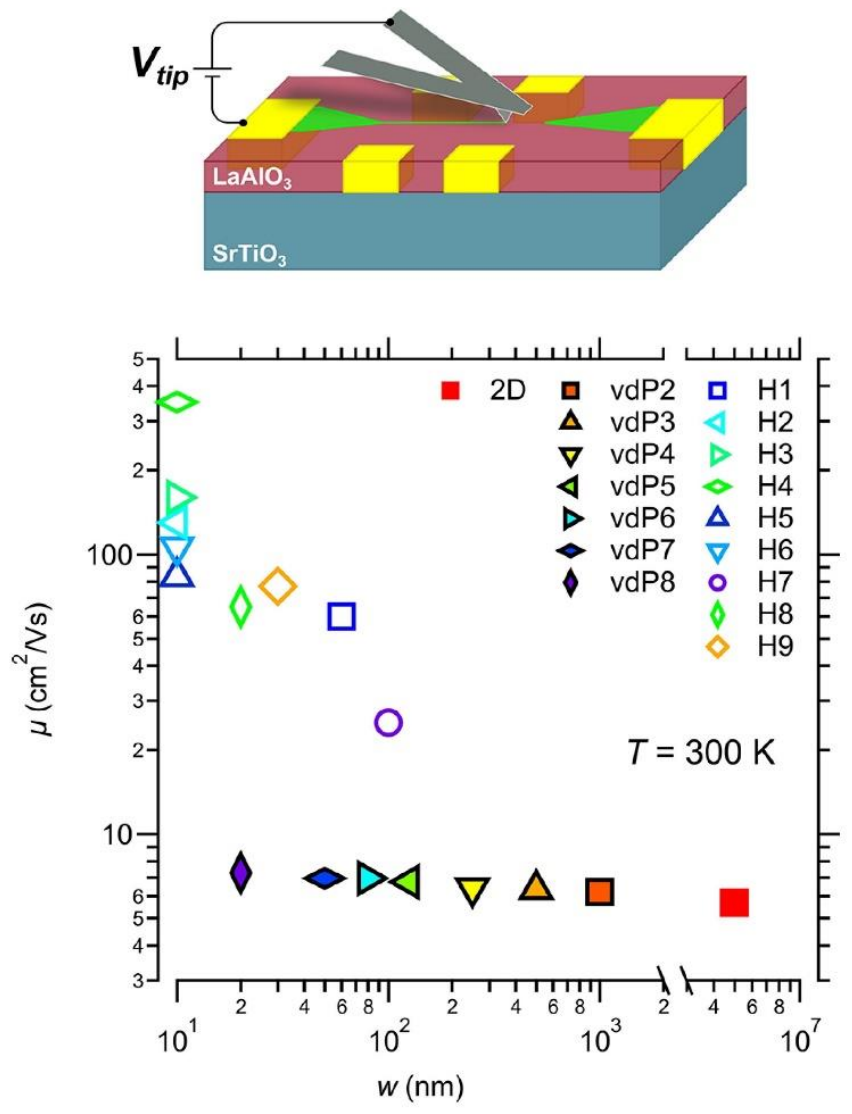

Figure 18 - Room temperature mobility $(\mu)$ as a function of the characteristic width $(w)$ of the conducting device formed at the LAO/STO interface using conducting atomic force microscopy writing. The mobilities are shown for van der Pauw devices (vdP), Hall-bar devices (H) and twodimensional confined conductivity at the intrinsic LAO/STO interface, with a large enhancement observed only for narrow Hall-bars where $w<100$ $\mathrm{nm}$. The inset shows a schematic of the writing process where a positively biased tip, scanned at the LAO surface, is used to create conductivity on the nanoscale at the LAO/STO interface. Reprinted with permission from reference [70]. Copyright 2013 American Chemical Society.

Cooling the Hall-bar structures also increased the mobility beyond what is usually observed in LAO/STO heterostructures, reaching a value exceeding $20,000 \mathrm{~cm}^{2} / \mathrm{Vs}$ at $20 \mathrm{~K}$ (see Figure 19). Curiously, deviations from the typical $\mu \propto T^{-2}$ behaviour were also observed (see Subsections 3.1 and 4.1). Although the origin of this mobility enhancement needs further investigation, the authors suggested that it is due a long-range coherence phenomenon occurring when electrons become confined in a quasi onedimensional structure.

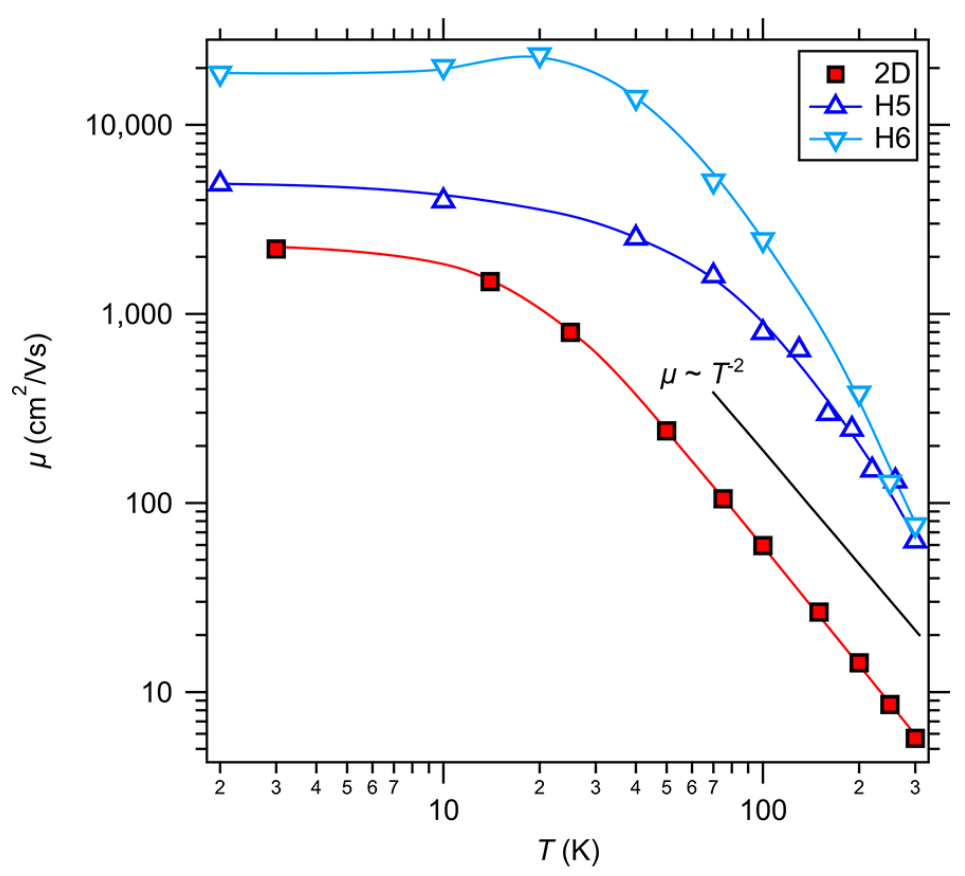

Figure 19 - Mobility $(\mu)$ as a function of temperature for two-dimensional confined conductivity at the LAO/STO interface as well as for conductivity formed in two Hall bar devices ( $\mathrm{H} 5$ and $\mathrm{H} 6$ ) with a width of $10 \mathrm{~nm}$. Reprinted with permission from reference [70]. Copyright 2013 American Chemical Society.

\section{Section 5: Mobility of Zinc Oxide: Bulk and thin-films}

\section{1 - Zinc Oxide (ZnO)}

Zinc oxide $(\mathrm{ZnO})$ is a semiconductor compound, which have attracted considerable interest in recent years. $\mathrm{ZnO}$ belongs to the class of II-VI semiconductors having the most stable B4 (wurtzite) structure. Moreover, has $\mathrm{ZnO}$ a direct band gap of $3.4 \mathrm{eV}$ and a high exciton binding energy of $60 \mathrm{meV}$, which is much higher than that of e.g. GaN (21-25 meV). Therefore, devices made from $\mathrm{ZnO}$ can operate more efficiently at high temperatures making it highly desirable for optoelectronic devices, such as light-emitting diodes, UV sources/sensors and room temperature lasing applications. The physical properties of $\mathrm{ZnO}$ are given in Table 3 [71]. 


\begin{tabular}{|l|c|c|}
\hline Physical property & Value & Ref. \\
\hline Molar mass & $81.408 \mathrm{~g} / \mathrm{mol}$ & PT \\
\hline Appearance & white & PT \\
\hline Density & $5.675 \mathrm{~kg} / \mathrm{m}^{3}$ & {$[19]$} \\
\hline Melting point & $1975^{\circ} \mathrm{C}$ & $\mathrm{PT}$ \\
\hline Boiling point & $2360^{\circ} \mathrm{C}$ & $\mathrm{PT}$ \\
\hline Refractive index & 1.614 & $\mathrm{PT}$ \\
\hline Direct and wide band gap & $\begin{array}{c}\text { direct band gap of } 3.44 \mathrm{eV} \text { at } \\
2 \mathrm{~K} \text { and } 3.37 \mathrm{eV} \text { at } 300 \mathrm{~K}\end{array}$ & {$[19]$} \\
\hline Large exciton binding energy & $60 \mathrm{meV}$ & {$[72]$} \\
\hline Piezoelectric constants & $1.34 \mathrm{C} / \mathrm{m}^{2}\left(\mathrm{e}_{33}\right),-0.57\left(\mathrm{e}_{31}\right)$ & {$[73]$} \\
\hline Effective mass & $0.3 \mathrm{me}$ & {$[74]$} \\
\hline $\begin{array}{l}\text { High-frequency dielectric } \\
\text { constant }\end{array}$ & 3.7 & {$[19]$} \\
\hline Static dielectric constant & 8.2 & {$[19]$} \\
\hline Lattice constant & $\mathrm{a}=3.2501 \AA$ and c $=5.2071 \AA$ & {$[75]$} \\
\hline Sound velocity & $6.09 \times 10^{5} \mathrm{~m} / \mathrm{s}$ & {$[19]$} \\
\hline Optical coupling constant & $3.9 \times 10^{11} \mathrm{eV} / \mathrm{m}$ & {$[19]$} \\
\hline
\end{tabular}

Table 3 - The physical parameters of $\mathrm{ZnO}$ adopted from the literature or the periodic table (PT).

There is a growing interest for the possibility of realizing active electronic devices exclusively made from transparent oxide materials, the so-called "transparent electronics" [76]. The discovery in 1997 of the oxide $\mathrm{CuAlO}_{2}$ with a high $p$-type (hole) conductivity [77] opened up for the first time for the possibility of combining transparent semiconducting $n$-type oxides with its $p$-type oxide counterpart. Having both $n$-and $p$-type oxides opened the door for a wide range of possible applications such as $p$ - $n$ junction diodes and UV-emitters. Another key device for realizing transparent circuits is the transparent field-effect transistor (TFET). For this application, one of the central challenges in the development of TFETs is getting a charge carrier channel with sufficiently high mobility. Once developed, these TFETs could in turn potentially be utilized in both existing display technologies and future integrated circuits [78]. When planning a thin film transistor layout and considering possible active layer material, amorphous semiconductors are generally preferred over their polycrystalline counterparts owing to their low processing temperature and uniformity of device characteristics. For example, when comparing the carrier mobility of e.g. amorphous-Si:H ( $\left.\mu \sim 1 \mathrm{~cm}^{2} / \mathrm{Vs}\right)$ with the crystalline one $\left(\mu \sim 200 \mathrm{~cm}^{2} / \mathrm{Vs}\right)$, it is clear that the mobility of the amorphous sample is still significantly lower. The low mobility is associated with the intrinsic nature of the difference in the chemical bonding. In contrast, a degenerate band conduction with relative large carrier mobility values $\left(\mu>10 \mathrm{~cm}^{2} / \mathrm{Vs}\right)$ is possible in amorphous oxide semiconductors containing post-transitionmetal cations. The electron transport in these materials is radically different from those of the covalent semiconductors. The conduction band bottom in oxide semiconductors is usually originating from hybridization between neighbouring atomic orbitals, which have direct overlap between them. Within these materials, doped ZnO plays a major role due to the quality of $\mathrm{ZnO}$ single-crystal substrates and films that one can achieve today. In these type of materials, a transparent amorphous oxide semiconductor from the ZnO system acting as the active channel in transparent thin-film transistors (TTFTs) show a relative high carrier mobility [79]. $\mathrm{ZnO}$ is also one of the systems which provide the exciting possibility of making thin-film transistors on flexible substrates with relatively high electron mobilities when compared with amorphous silicon or organic semiconductors [80]. In the following, we will review the carrier mobility of electrons in bulk conducting $\mathrm{ZnO}$ followed by discussion on the mobility of its derivates. Unlike STO, thin films of ZnO plays an important technological role and we have therefore devoted a part of Section 5 on this topic. The subsequent Section 6 will cover the mobility of twodimensional electron gases in $\mathrm{ZnO}$-based systems.

\section{2 - Carrier mobility in bulk ZnO}

Typical Hall mobility data measured on bulk conducting $n$-type $\mathrm{ZnO}$ show an increase of the carrier mobility from about $190 \mathrm{~cm}^{2} / \mathrm{Vs}$ at room temperature to a maximal value of $2000 \mathrm{~cm}^{2} / \mathrm{Vs}$ as the temperature is decreased to $50 \mathrm{~K}$ (see Figure 20).

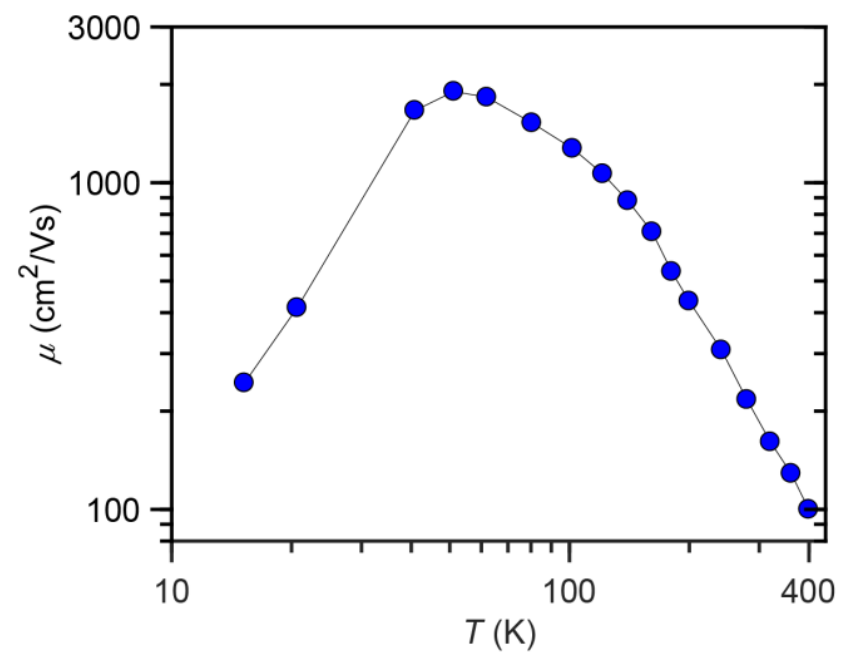

Figure 20 - Hall mobility as a function of temperature for bulk $\mathrm{ZnO}$. The data is obtained from reference [81].

The electron mobility of $2000 \mathrm{~cm}^{2} / \mathrm{Vs}$ at about $50 \mathrm{~K}$ is the highest reported value for bulk conducting $\mathrm{ZnO}$. In Table 4 we show the mobility of bulk conducting $\mathrm{ZnO}$ produced by different methods. As one can see from this table, the mobility of $\mathrm{ZnO}$ seems to be sensitive to the fabrication method but with an overall average value of approximately $180 \mathrm{~cm}^{2} / \mathrm{Vs}$ at room temperature.

\begin{tabular}{|l|c|c|}
\hline Preparation Method & Electron mobility $\left(\mathbf{c m}^{2} / \mathbf{V s}\right)$ & Ref. \\
\hline Vapor-phase & 1900 at $50 \mathrm{~K}$ & {$[81]$} \\
\hline Hydrothermal & 156 at $300 \mathrm{~K}, 19$ at $77 \mathrm{~K}$ & {$[82]$} \\
\hline Chemical vapor transport (CVT) & $\begin{array}{c}197-215 \text { at } 300,560-580 \\
\text { at } 80 \mathrm{~K}\end{array}$ & {$[83]$} \\
\hline Hydrothermal & 200 at $300 \mathrm{~K}$ & {$[84]$} \\
\hline HT ZnO wafers & 300 at $300 \mathrm{~K}$ & {$[85]$} \\
\hline high-pressure melt grown (HPM) & 203 at 300 K & {$[86]$} \\
\hline hydrothermal (HYD) & 134 at 300 K & {$[86]$} \\
\hline seeded-CVT method & 205 at 300 K & {$[86]$} \\
\hline seeded vapor-phase (SVP) & 205 at 300 K & {$[87]$} \\
\hline Flux Bridgmann Methode & 124 at 300 K & {$[88]$} \\
\hline
\end{tabular}

Table 4 - The mobility of bulk $\mathrm{ZnO}$ fabricated by different techniques and measured at room temperature.

For many applications, such as light-emitting diodes, it is necessary to obtain both the $n$-type and the $p$-type materials. Realizing bulk conducting $p$-type $\mathrm{ZnO}$, however, still poses a challenge, which remains a major hindrance for future applications. The $p$-type doping is difficult to achieve in $\mathrm{ZnO}$ [89] due to the existence of background $n$-type dopants including $\mathrm{H}$ 
impurities [90], O vacancies [91] and $\mathrm{Zn}$ interstitials [92]. Despite the difficulties in obtaining bulk $p$-type $\mathrm{ZnO}$, several laboratories have demonstrated thin film $p$-type $\mathrm{ZnO}$ using group $\mathrm{V}$ (N [93], $\mathrm{P}$ [94], As [95], and $\mathrm{Sb}$ [96]) and group I (Li [97]) elements. Co-doping with two potential acceptors ( $\mathrm{N}$ and $\mathrm{As}$ ) [98] or acceptor and donor ( $\mathrm{N}$ and $\mathrm{Al}$ ) [99] have also been studied. The room temperature hole mobility of these epitaxial thin films was slightly higher than $7.7 \mathrm{~cm}^{2} / \mathrm{Vs}$ and achieved by carful controlling of the residual impurity densities. Although this hole mobility is not as high as in $p$-type GaN it still represents a significant advantage towards utilizing $\mathrm{ZnO}$ in future applications. The hole transport characteristics are calculated using the "relaxation time approximation" as a function of temperature [19]. As mentioned in Section 2.2.2, the acoustic phonon scattering is the most important mechanisms in $p$-type $\mathrm{ZnO}$, limiting the hole mobility over a wide range of temperature. For temperatures above $300 \mathrm{~K}$, the polar optical phonon scattering mechanism is the dominant factor (see Figure 21).

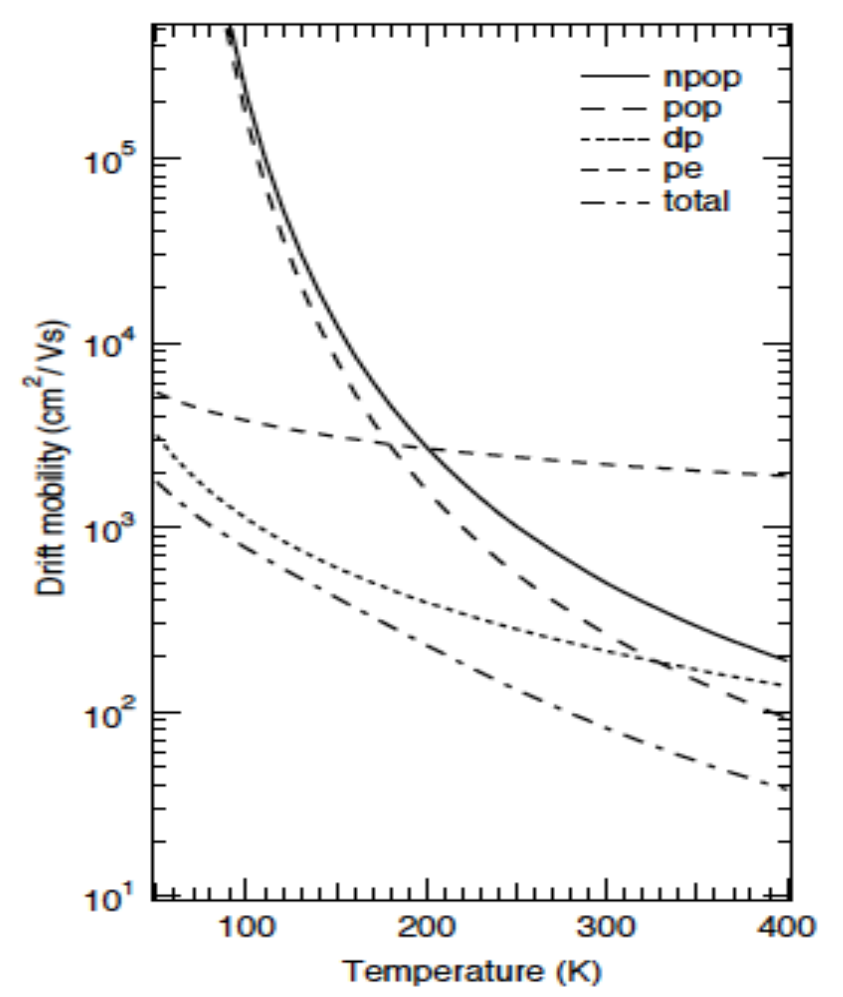

Figure 21 - Theoretical scattering model for electron mobility of $p$-type $\mathrm{ZnO}$ thin films. Here, the different scatting contributions in $\mathrm{ZnO}$ are: acoustic (dp) and optical phonons (npop), polar optical phonon (pop), piezoelectric deformations (pe). The figure is adapted from reference [19]. Copyright 2006 The Japan Society of Applied Physics.

\section{3 - Carrier mobility in $\mathrm{ZnO}$ based thin films}

The most widely studied transparent conductive oxide (TCO) thin film material is Sn-doped indium oxide (ITO), where carrier densities are exceeding $10^{21} \mathrm{~cm}^{-3}$ and resistivities are below $10^{-5} \Omega \mathrm{cm}$. However, the high cost and scarceness of indium has stimulated the search for a replacement material. Recent work has therefore been concentrated on finding alternative materials including promising candidates within the binary oxides $\mathrm{ZnO}, \mathrm{SnO}_{2}$ [100], $\mathrm{CdO}$ [101], and $\mathrm{Ga}_{2} \mathrm{O}_{3}$ [102], and within multi-component oxides such as indium-gallium-zinc-oxide (IGZO) [103] and cubic spinel oxide compounds such as $\mathrm{Cdln}_{2} \mathrm{O}_{4}[104]$ and $\mathrm{SnZn}_{2} \mathrm{O}_{4}$
[105]. Such alternatives have been reviewed by Ellmer [106]. Here, obtaining a high carrier mobility at room temperature is crucial as it allows for a large conductivity while not sacrificing the transparency by adding additional dopants.

To date, the highest room temperature mobility observed for $\mathrm{ZnO}$ was in a bulk single crystal with values approaching $\mu \sim 200 \mathrm{~cm}^{2} / \mathrm{Vs}$ and carrier density $n \sim 1 \times 10^{17} \mathrm{~cm}^{-3}$. Doping thin film ZnO with either Al [107] or Ga [108], was found to increase the carrier density while the resistivity reduced to $10^{-4} \Omega \mathrm{cm}$. Introducing other potential dopants into the $\mathrm{ZnO}$ thin film have previously been studied for e.g. In-doped ZnO (IZO) [109] and Inand Ga-doped ZnO (IGZO) [110]. The Al-doped ZnO (AZO) has been considered to be one of the best candidates as a transparent conductor due to the relative low cost, the natural abundance of $\mathrm{Zn}$ and $\mathrm{Al}$ as well as the good optical transmission of this compound in the visible range. However, these materials are still facing the following challenges [103]: (1) their room temperature resistivity still remains high, and (2) the transport properties are strongly dependent on film thickness. For these films the additional dopants furthermore reduce the mobility to a relatively low range of $\mu \sim 30-50 \mathrm{~cm}^{2} / \mathrm{Vs}$ for a carrier density of around $n \sim 10^{20} \mathrm{~cm}^{-3}$, primarily because of ionized impurity scattering (see Figure 22). In Figure 22 we show a representative collection of different compositions with their respective mobility and carrier densities measured at room temperature. Here, the carrier density and mobility at room temperature are between $n$ $\sim 10^{14}-10^{18} \mathrm{~cm}^{-3}$ and $\mu \sim 70-150 \mathrm{~cm}^{2} / V s$, respectively.

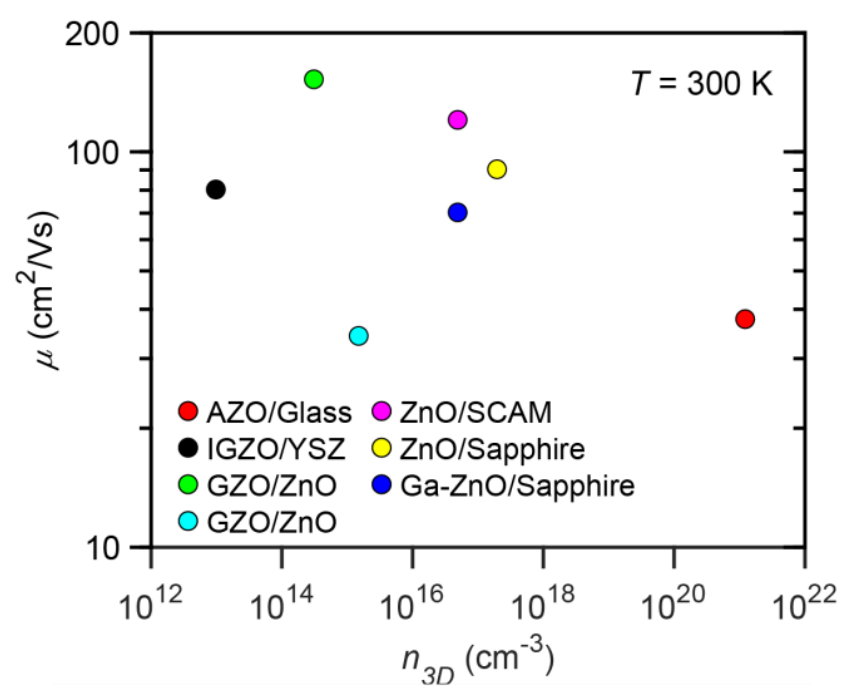

Figure 22 - The dependence of the room temperature electron mobility, $\mu$, on the corresponding bulk carrier density, $n_{3 \mathrm{D}}$, for bulk conducting $\mathrm{ZnO}$ based systems. The data is obtained from: Al-doped ZnO (AZO)/Glass [111]; In- and Ga-codoped ZnO (IGZO) on yttrium-stabilied zirconium (YSZ) [79]; Ga-doped ZnO (GZO)/ZnO [112]; ZnO/SCAM [109]; ZnO/Sapphire [109], Gadoped ZnO/Sapphire (Ga-ZnO/Sapphire) [108].

While traditional TCOs are often highly crystalline, a new class of materials, amorphous indium-doped $\mathrm{ZnO}$ (a-IZO) with an unusually high electron mobility, $\mu \sim 54 \mathrm{~cm}^{2} / \mathrm{Vs}$ and carrier density of $n \sim 1.3 \times 10^{20} \mathrm{~cm}^{-3}$ was recently reported by Leenheer et al. [111]. A general observation of these type of materials is that the mobility seems to depend on the carrier density, which in turn can be tuned by annealing in oxygen. 


\section{Section 6: Mobility of Zinc Oxide: Two- dimensional electron gases}

\section{1 - Mobility in ZnO based heterostructures}

Much effort has been made in designing two-dimensional electron gases in $\mathrm{ZnO}$-based systems. The first report on high carrier mobility at the (0001)-oriented $\mathrm{Mg}_{x} \mathrm{Zn}_{1-x} \mathrm{O} / \mathrm{ZnO}$ heterostructures was realized in 2007 using Pulsed Laser Deposition (PLD) by Tsukazaki et al. [11]. The $\mathrm{Mg}_{x} \mathrm{Zn}_{1-x} \mathrm{O}$ layer in their heterostructure acts as a potential barrier for the twodimensional electron gas (2DEG) in the adjacent $\mathrm{ZnO}$ layer by gradually adjusting the Mg content. In this study, the authors found that the carrier density and the mobility depended on the amount of magnesium doping (i.e. $x$ ). The authors demonstrated sheet carrier densities of $n_{s} \sim 0.66 \times 10^{12}$ and $3.7 \times 10^{12} \mathrm{~cm}^{-2}$ with electron mobilities of $\mu \sim 5500$ and $2700 \mathrm{~cm}^{2} / \mathrm{Vs}$ at $1 \mathrm{~K}$ for $x=0.15$ and $x=0.2$, respectively. Although PLD provided the initial means to achieve a high interfacial mobility, molecular-beam epitaxy (MBE) now serves as the thin-film growth method of choice to achieve exceptional high purity and cleanliness of $\mathrm{MgZnO}$ interfaces. Soon after the initial results obtained with PLD, Tsukazaki et al. showed that electron mobilities higher than the one obtained by PLD could been attained in $\mathrm{Mg}_{x} \mathrm{Zn}_{1-x} \mathrm{O} / \mathrm{ZnO}$ heterostructures grown by $\mathrm{MBE}$ [112] i.e. electron mobility in the order of $14,000 \mathrm{~cm}^{2} / \mathrm{Vs}$. The doubling of the electron mobility of samples obtained by MBE as compared to PLD grown sample was attributed to the growth direction and strain [112]. Further improvement of the growth conditions resulted in even higher mobility values of $\mathrm{MgZnO} / \mathrm{ZnO}$ heterostructures grown by MBE with an electron mobility exceeding $180,000 \mathrm{~cm}^{2} / \mathrm{Vs}$ [113]. This high electron mobility allowed observation of the fractional quantum Hall effect for the first time in oxides. The observation underlined the cleanliness and extremely smoothness of the $\mathrm{MgZnO} / \mathrm{ZnO}$ interface as they both are prerequisites for the observation of this effect.

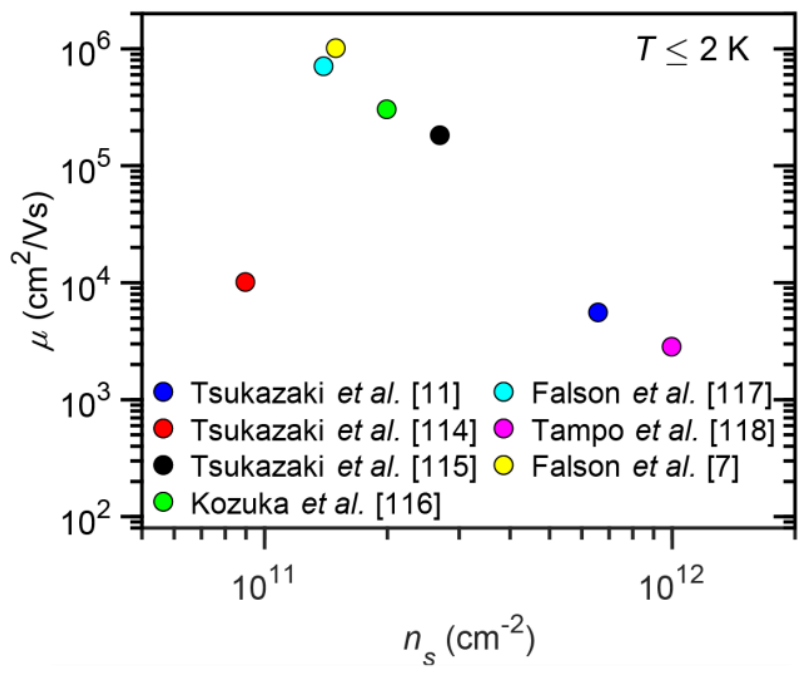

Figure 23 - The dependence of the low-temperature electron mobility, $\mu$, on the sheet carrier density, $n_{s}$, for confined ZnO-based systems. The data is obtained from: $\mathrm{Mg}_{0.15} \mathrm{Zn}_{0.85} \mathrm{O} / \mathrm{ZnO}, \mathrm{PLD}$, Tsukazaki, et al. [11]; $\mathrm{Mg}_{0.08} \mathrm{Zn}_{0.92} \mathrm{O} / \mathrm{ZnO}, \mathrm{MBE}$, Tsukazaki et al. [112]; MgZnO/ZnO, MBE, Tsukazaki et al. [113]; MgZnO/ZnO, MBE, Kozuka et al. [114]; $\mathrm{Mg}_{0.01} \mathrm{Zn}_{0.99} \mathrm{O} / \mathrm{ZnO}, \mathrm{MBE}$, Falson et al. [115]; ZnMgO/ZnO, Radical source
MBE, Tampo et al. [116]; MgZnO/ZnO, Ozon-molecular MBE, Falson et al. [7].

Impressively, this was the first demonstration of fractional quantum Hall effect in an ionic material with much stronger correlation between electrons compared to conventional semiconductor heterostructures [113]. Further improvements of the electron mobility to a value of 300,000 $\mathrm{cm}^{2} / \mathrm{Vs}$ and a magnetic field up to $26 \mathrm{~T}$ at $40 \mathrm{mK}$ made it possible to further study lower filling factor fractional quantum states in oxide materials [114]. This was due to a reduced charge carrier density compared to the samples studied earlier [113]. Additional increase of the mobility to a value of $700,000 \mathrm{~cm}^{2} / \mathrm{Vs}$ for the $\mathrm{Mg}_{0.01} \mathrm{Zn}_{0.99} \mathrm{O} / \mathrm{ZnO}$ heterostructures grown by $\mathrm{MBE}$ was achieved by a reduction of impurity levels associated with the use of pure distilled ozone and avoiding interface roughness scattering [115]. The dependence of electron mobility with 2DEG sheet carrier density measured at temperatures below $2 \mathrm{~K}$ is shown in Figure 23.

The electron mobility values shown in Figure 23 represent the highest reported mobility values for different $\mathrm{ZnO}$-based heterostructures. The continuous improvement in the mobility over the years stems from the refinement the MBE growth conditions while using ozone to reduce the residual impurity content. The electron mobility improvement is, however, also a result of carefully controlling the carrier density with a lower $\mathrm{Mg}$ content of samples. Care was likewise taken to prepare single epitaxial layers using MBE on a ZnO single crystal substrates (Fabricated by hydrothermal method), tuning the $\mathrm{Mg}$ content and improving the purity of the $\mathrm{Zn}$ source material. In addition, the use of ozone as an oxygen source offers the benefit of an expanded temperature window for uniform growth. More details of the preparation method can be found in reference [7]. This above mentioned careful procedure enabled a record-high electron mobility exceeding a staggering $1,000,000 \mathrm{~cm}^{2} / \mathrm{Vs}$ [7]. In general, these high electron mobilities occur only for a narrow range of charge densities. Figure 24 show the evolution of electron mobility improvements as a function of sheet carrier density over the years. It is evident from Figure 24 that although the electron density continuously was improved, two distinct scattering mechanisms are limiting the electron mobility depending on the carrier density regime. For low carrier densities, charged impurity scattering is dominating $(\mu \propto n$, see Section 2.1) while interface/alloy scattering limits the electron mobility at higher electron densities ( $\mu \propto n^{-3 / 2}$, see Section 2.4). 


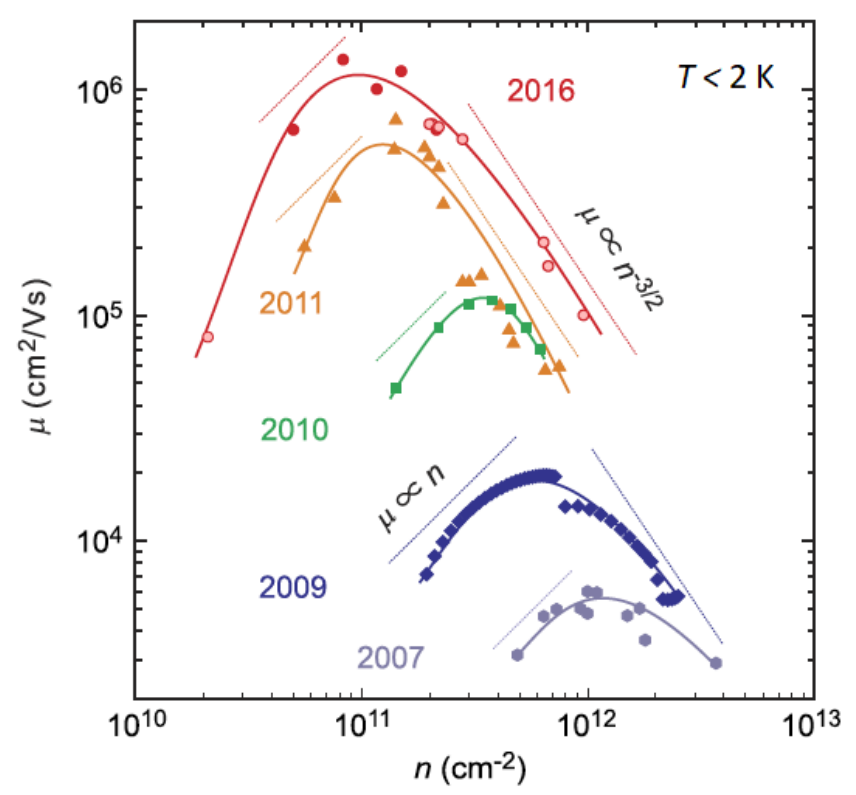

Figure 24 - The improvements in electron mobility $(\mu)$ over the years as function of sheet carrier density $(n)$ for temperatures $T \leq 2 \mathrm{~K}$. To guide the eye the mobility scaled by $\mu \propto n$ and $\mu \propto n^{-3 / 2}$ are indicated at each data set corresponding to charged impurity scattering and interface/alloy scattering, respectively. The figure shows the evolution of $\mu$ over the years at 2007 [11], 2009 [117], 2010 [113], 2011 [115] and 2016 [7]. The figure is adapted from reference [7].

\section{Section 7: Beyond strontium titanate and Zinc Oxide}

Although STO and ZnO exhibit the largest electron mobilities in oxides at low temperature, a large number of oxide structures beyond these have a comparable or even larger mobility at room temperature. The room temperature electron mobility versus carrier density for thin films of key oxide compounds such as $\mathrm{SnO}_{2}$ [118], $\mathrm{TiO}_{2}$ [119], $\mathrm{ZnO}$ [120], $\mathrm{Ga}_{2} \mathrm{O}_{3}$ [121], $\mathrm{In}_{2} \mathrm{O}_{3}$ [122], $\mathrm{CdO}$ [123], $\mathrm{BaSnO}_{3}[124]$ and $\mathrm{SrRuO}_{3}$ [125] are summarized in Figure 25 .

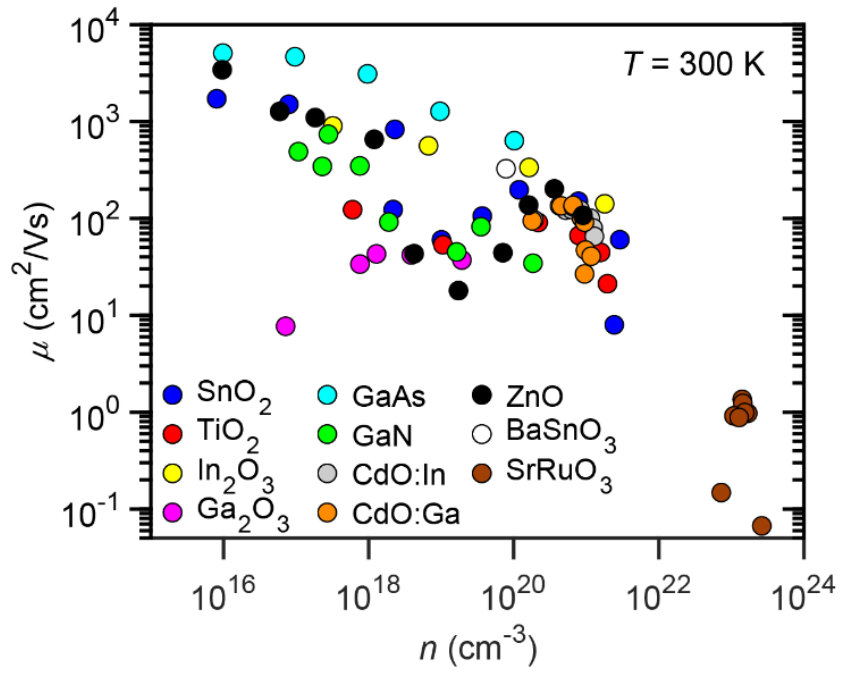

Figure 25 - The dependence of room-temperature electron mobility, $\mu$, on the carrier density, $n$, for different bulk conducting oxide-based systems either as a single crystal or as a thin film. The data is taken from: $\mathrm{SnO}_{2}$ [118], $\mathrm{TiO}_{2}$ [119], $\mathrm{ZnO}$ [120], $\mathrm{Ga}_{2} \mathrm{O}_{3}$ [121], $\mathrm{In}_{2} \mathrm{O}_{3}$ [122], $\mathrm{CdO}: \mathrm{Ga}, \operatorname{In}$ [123], $\mathrm{GaN}$ [126], $\mathrm{GaAs}$ [127], $\mathrm{BaSnO}_{3}$ [124] and $\mathrm{SrRuO}_{3}$ [125].

For comparison, we have included additional data for traditional high mobility semiconductors such as GaN [126] and GaAs [127]. Another type of perovskite oxides that recently have gained attention is the Alkalineearth stannates with the generic composition $\mathrm{ASnO}_{3}(\mathrm{~A}=\mathrm{Ca}, \mathrm{Sr}$, and $\mathrm{Ba})$. Among $\mathrm{ASnO}_{3}$, the cubic $\mathrm{BaSnO}_{3}$, which is a $n$-type semiconductor stable at temperatures up to $1000{ }^{\circ} \mathrm{C}$, has been intensively investigated [128]. Earlier work showed that the electron mobility of thin film and polycrystalline doped $\mathrm{BaSnO}_{3}$ is relatively low $\left(\mu<1 \mathrm{~cm}^{2} / \mathrm{Vs}\right)$ [128]. However, $\mathrm{BaSnO}_{3}$ single crystals can now display electron mobilities of $\mu \sim$ $320 \mathrm{~cm}^{2} / \mathrm{Vs}$ [124] at room temperature with a doping level of $8 \times 10^{19} \mathrm{~cm}^{-3}$. Therefore, there has been high demand for the growth of high-quality single crystals of doped $\mathrm{BaSnO}_{3}$. Raghavan et al. reported MBE-grown $(\mathrm{Ba}, \mathrm{La}) \mathrm{SnO}_{3}$ thin films on $\mathrm{PrScO}_{3}(110)$ substrates with room temperature mobilities of $\mu \sim 150 \mathrm{~cm}^{2} / \mathrm{Vs}$ by use of preoxidized $\mathrm{Sn}$ as a precursor [129]. This is the highest-known electron mobility in doped $\mathrm{BaSnO}_{3}$ thin films at room temperature. The previously reported mobility values of $\mathrm{BaSnO}_{3}$ and doped $\mathrm{BaSnO}_{3}$ on different substrates is presented in Figure 26. In addition, the mobility was enhanced to around $300 \mathrm{~cm}^{2} / \mathrm{Vs}$ at $50 \mathrm{~K}$ by varying the carrier density in an electric-double-layer setup where an electric field was applied to $\mathrm{a} \mathrm{BaSnO}_{3}$ thin film through an ionic liquid [130]. A more comprehensive review on the high electron mobility of doped $\mathrm{BaSnO}_{3}$ for TCOs can be found in reference [131]. 


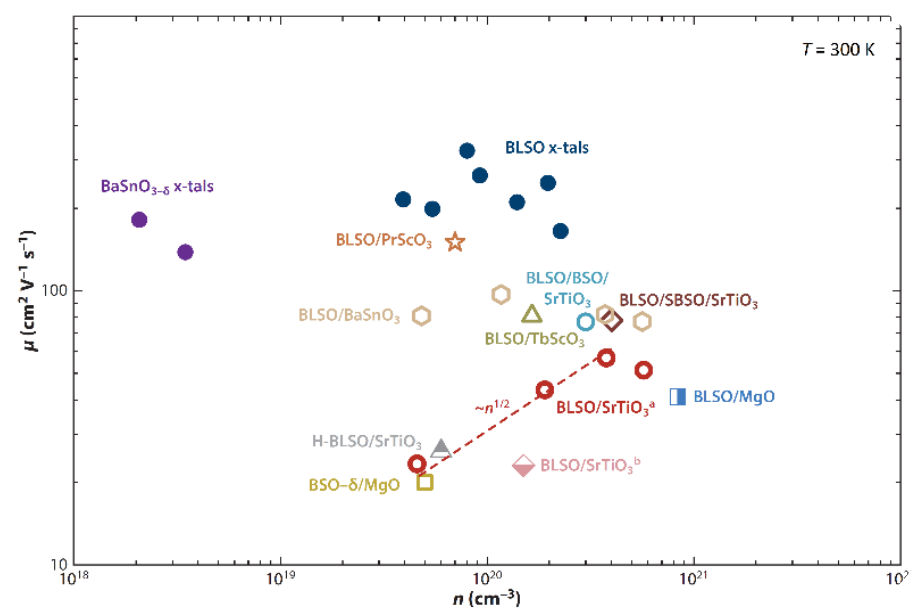

Figure 26 - The dependence of room-temperature ( $T=300 \mathrm{~K}$ ) electron mobility, $\mu$, on the carrier density, $n$, for La-doped $\mathrm{BaSnO}_{3}$. Republished with permission of Annual Reviews, from reference [131]; permission conveyed through Copyright Clearance Center, Inc.

\section{1 - Confined systems beyond STO- and ZnO-based heterostructures}

Integrating two-dimensional oxide electron gases with semiconductors opens prospects of new functionalities arising from the potential of coupling the 2DEG carriers with the semiconductor. This was studied by Kornblum et al. where the authors prepared (001) $\mathrm{GdTiO}_{3} / \mathrm{SrTiO}_{3}$ (GTO/STO) heterostructures on (001) GaAs [132]. The sheet carrier density seems to be independent on the temperature with a carrier density value of $\sim 4 \times 10^{14} \mathrm{~cm}^{-2}$ and electron mobility of $\sim 4 \mathrm{~cm}^{2} / \mathrm{Vs}$ from $60 \mathrm{~K}$ to $300 \mathrm{~K}$.

Another successful demonstration is the formation of 2DEG at the interface between a-LAO and $\mathrm{TiO}_{2}$ (Anatase) as well as $\mathrm{TiO}_{2}$ (Rutile) substrates [133]. The $\mathrm{TiO}_{2}$ (R)-based heterostructure presents a comparable carrier density to the intensively investigated system of aLAO/STO, but with a much lower mobility, i.e. $\sim 77 \mathrm{~cm}^{2} / \mathrm{Vs}$ at $30 \mathrm{~K}$, and in contrast, the a-LAO/TiO $(\mathrm{R})$ gives rise to a low mobility of $\sim 24.5 \mathrm{~cm}^{2} / \mathrm{Vs}$ at $2 \mathrm{~K}$. The authors of this work concluded that the interfacial conduction comes from redox reactions, and that the differences between the materials systems result mainly from variations in the activation energies for the diffusion of oxygen vacancies at substrate surfaces.

In another recent work, a new 2DEG system at the interface between a Mott insulator, $\mathrm{LaTiO}_{3}$, and a band insulator, $\mathrm{KTaO}_{3}$ was reported [134]. A metallic conductive interface was observed from $2 \mathrm{~K}$ to $300 \mathrm{~K}$. The mobility of these interfaces seems to be independent of the thickness of $\mathrm{LaTiO}_{3}$ and found to be $\sim 10 \mathrm{~cm}^{2} / \mathrm{Vs}$ at $300 \mathrm{~K}$ and $\sim 100 \mathrm{~cm}^{2} / \mathrm{Vs}$ at $2 \mathrm{~K}$. This study provides alternative oxide heterostructures that host $2 \mathrm{DEGs}$.

There have also been theoretical studies for potential confined electronic systems where the conduction bands are comprised of highly dispersive non-degenerate $s$ orbitals. In a recent work, first-principles density functional theory calculations were used on oxide materials with $s$-like conduction bands [135]. Here, the interface studied was between nonpolar perovskite $\mathrm{BaSnO}_{3}$ and polar perovskite $\mathrm{LaScO}_{3}$ stacked along the [001] direction. $\mathrm{BaSnO}_{3}$ and $\mathrm{LaScO}_{3}$ single crystals are perovskite with band gaps of $\sim 3 \mathrm{eV}$ and $5.7 \mathrm{eV}$, respectively [136]. The conduction band electrons were found to reside in the highly dispersive $\mathrm{Sn}-5 \mathrm{~s}$ orbitals, which have a large band width and a low effective mass. The result of the calculation indicate that the predicted $2 D E G$ is expected to be highly mobile even at room temperature due to the reduced electron-phonon scattering via the inter-band scattering channel [135].

In another theoretical work, Wang et al. attempted to substitute STO with other nonpolar perovskite oxide whose lowest energy conduction bands are comprised of $s$ or $p$ orbitals [136]. The authors used first-principles electronic structure calculations and explored the possibility of producing a high-mobility 2DEG in the following two systems: $\mathrm{LaAlO}_{3} / \mathrm{SrGeO}_{3}$ and $\mathrm{LaGaO}_{3} / \mathrm{BaSnO}_{3}$ i.e. with $\mathrm{SrGeO}_{3}$ and $\mathrm{BaSnO}_{3}$ as substrate materials [136]. Under the assumption of constant scattering time approximation, the calculations indicated that the room temperature electron mobility and the electrical conductivity of $\mathrm{LaAlO}_{3} / \mathrm{SrGeO}_{3}$ and $\mathrm{LaGaO}_{3} / \mathrm{BaSnO}_{3}$ systems should be nearly 2.5 and 2.0 times larger, respectively, than in the LAO/STO 2DEG [136]. These theoretical predictions are however yet to be demonstrated experimentally.

\section{Section 8: Perspectives}

The majority of oxides still suffers from a low carrier mobility due to structural imperfections and a large number of impurities. However, the recent progress in achieving high carrier mobility in oxides and oxide heterostructures has led to improvements in, e.g., transparent conductors and realization of quantum phenomena in oxides. In particular, material systems based on STO and ZnO have undergone a large development and now reach electron mobilities exceeding 100,000 and $1,000,000 \mathrm{~cm}^{2} / \mathrm{Vs}$, respectively. Such high electron mobilities open up various avenues:

1) Many oxides exhibit a large range of remarkable properties. It is of high interest to achieve new functionalities by combining these properties with the quantum phenomena that can be observed in high-mobility materials.

2) Many properties in functional oxides stem from intentional or unintentional defects, but the properties can be hard to control and reproduce as the purity of oxides generally falls much below that of silicon. In the process of achieving high carrier mobilities, unintentional impurities are often reduced drastically, which allows for a better control by introducing intentional impurities in a controlled manner into a clean system.

3) Oxides are promising for various applications, which benefit from a high carrier mobility. These include thermoelectricity due to the chemical stability and low price tag of oxides as well as use as transparent conductors where the high band gap in oxides assures transparency in the visible range. Oxides may also be used in, e.g., magnetoresistive devices where the large positive magnetoresistance intrinsically found in some oxides may be combined with a positive magnetoresistance stemming from the extraordinary magnetoresistance [56].

\section{Acknowledgements}

F. Trier acknowledges support by research grant VKR023371 (SPINOX) from VILLUM FONDEN. 


\section{References}

[1] Umansky V, Heiblum M, Levinson $Y$, Smet J, Nübler J and Dolev M 2009 MBE growth of ultra-low disorder 2DEG with mobility exceeding $35 \times 10^{6} \mathrm{~cm}^{2} / \mathrm{Vs} J$. Cryst. Growth 311 1658-61

[2] Klitzing K v, Dorda G and Pepper M 1980 New method for high-accuracy determination of the finestructure constant based on quantized Hall resistance Phys. Rev. Lett. 45494

[3] Tsui D C, Stormer H L and Gossard A C 1982 Twodimensional magnetotransport in the extreme quantum limit Phys. Rev. Lett. 481559

[4] Kroemer H 2001 Nobel lecture: quasielectric fields and band offsets: teaching electrons new tricks Rev. Mod. Phys. 73783

[5] Ohtomo A and Hwang $\mathrm{H}$ Y 2004 A high-mobility electron gas at the $\mathrm{LaAlO}_{3} / \mathrm{SrTiO}_{3}$ heterointerface Nature 427 423-426

[6] Chen Y Z, Bovet N, Trier F, Christensen D V, Qu F M, Andersen N H, Kasama T, Zhang W, Giraud R, Dufouleur J, Jespersen T S, Sun J R, Smith A, Nygård J, Lu L, Büchner B, Shen B G, Linderoth S and Pryds N 2013 A high-mobility two-dimensional electron gas at the spinel/perovskite interface of $\mathrm{y}^{-} \mathrm{Al}_{2} \mathrm{O}_{3} / \mathrm{SrTiO}_{3} \mathrm{Nat}$. Commun. 41371

[7] Falson J, Kozuka Y, Uchida M, Smet J H, Arima T, Tsukazaki A and Kawasaki M 2016 MgZnO/ZnO heterostructures with electron mobility exceeding 1 $\times 10^{6} \mathrm{~cm}^{2} /$ Vs Sci. Rep. 626598

[8] Harrang J P, Higgins R J, Goodall R K, Jay P R, Laviron $M$ and Delescluse $P 1985$ Quantum and classical mobility determination of the dominant scattering mechanism in the two-dimensional electron gas of an AlGaAs/GaAs heterojunction Phys. Rev. B 328126

[9] Trier F, Prawiroatmodjo G E D K, Zhong Z, Christensen D V, von Soosten M, Bhowmik A, Lastra J M G, Chen Y, Jespersen T S and Pryds N 2016 Quantization of Hall Resistance at the Metallic Interface between an Oxide Insulator and $\mathrm{SrTiO}_{3}$ Phys. Rev. Lett. 117096804

[10] Caviglia A D, Gariglio S, Cancellieri C, Sacépé B, Fête A, Reyren N, Gabay M, Morpurgo A F and Triscone JM 2010 Two-Dimensional Quantum Oscillations of the Conductance at $\mathrm{LaAlO}_{3} / \mathrm{SrTiO}_{3}$ Interfaces Phys. Rev. Lett. 105236802

[11] Tsukazaki A, Ohtomo A, Kitamura T, Ohno Y, Ohno H and Kawasaki M 2007 Quantum Hall Effect in Polar Oxide Heterostructures Science 315 1388-91

[12] Hamaguchi C 2010 Basic semiconductor physics (Berlin, Heidelberg: Springer Berlin Heidelberg)

[13] Ellmer K, Klein A and Rech B 2008 Transparent conductive zinc oxide: basics and applications in thin film solar cells (Berlin: Springer)

[14] Bass S J 1979 Silicon and germanium doping of epitaxial gallium arsenide grown by the trimethylgallium-arsine method J. Cryst. Growth $\mathbf{4 7}$ 613-8

[15] Dingle R, Störmer H L, Gossard A C and Wiegmann W 1978 Electron mobilities in modulation-doped semiconductor heterojunction superlattices Appl. Phys. Lett. 33665

[16] Low F E and Pines D 1955 Mobility of slow electrons in polar crystals Phys. Rev. 98414

[17] Peeters F M, Wu X and Devreese J T 1988 Exact and approximate results for the mass of a twodimensional polaron Phys. Rev. B 37933

[18] Verma A, Kajdos A P, Cain T A, Stemmer S and Jena D 2014 Intrinsic Mobility Limiting Mechanisms in Lanthanum-Doped Strontium Titanate Phys. Rev. Lett. 112216601

[19] Makino T, Tsukazaki A, Ohtomo A, Kawasaki M and Koinuma H 2006 Hole transport in p-type ZnO Jpn. J. Appl. Phys. 456346

[20] Özgür ü., Alivov Y I, Liu C, Teke A, Reshchikov M A, Doğan S, Avrutin V, Cho S-J and Morkoç H 2005 A comprehensive review of $\mathrm{ZnO}$ materials and devices J. Appl. Phys. 98041301

[21] Mikheev E, Himmetoglu B, Kajdos A P, Moetakef P, Cain T A, Van de Walle C G and Stemmer S 2015 Limitations to the room temperature mobility of twoand three-dimensional electron liquids in $\mathrm{SrTiO}_{3} \mathrm{Appl}$. Phys. Lett. 106062102

[22] Fu H, Reich K V and Shklovskii B I 2016 Surface roughness scattering in multisubband accumulation layers Phys. Rev. B 93 
[23] Tufte O N and Chapman P W 1967 Electron mobility in semiconducting strontium titanate Phys. Rev. 155 796

[24] Frederikse H P R and Hosler W R 1967 Hall Mobility in $\mathrm{SrTiO}_{3}$ Phys. Rev. 161822

[25] Koonce C S, Cohen M L, Schooley J F, Hosler W R and Pfeiffer E R 1967 Superconducting Transition Temperatures of Semiconducting $\mathrm{SrTiO}_{3}$ Phys Rev 163 380-390

[26] Sulpizio J A, Ilani S, Irvin P and Levy J 2014 Nanoscale Phenomena in Oxide Heterostructures Annu. Rev. Mater. Res. 44 117-49

[27] Blazey K 1971 Optical Absorption Edge of $\mathrm{SrTiO3}$ around 105-K Phase Transition Phys. Rev. Lett. 27 146-

[28] van Benthem K, Elsässer C and French R H 2001 Bulk electronic structure of $\mathrm{SrTiO}_{3}$ : Experiment and theory J. Appl. Phys. 906156

[29] Gogoi P K, Sponza L, Schmidt D, Asmara T C, Diao C, Lim J C W, Poh S M, Kimura S, Trevisanutto P E, Olevano $V$ and Rusydi A 2015 Anomalous excitons and screenings unveiling strong electronic correlations in $\mathrm{SrTi} 1-\mathrm{x} \mathrm{Nb} \times \mathrm{O} 3(0 \leq \mathrm{x} \leq 0.005)$ Phys. Rev. B 92

[30] Furuta T and Miura K 2010 First-principles study of ferroelectric and piezoelectric properties of tetragonal $\mathrm{SrTiO} 3$ and $\mathrm{BaTiO} 3$ with in-plane compressive structures Solid State Commun. 150 2350-3

[31] Christen H-M, Mannhart J, Williams E J and Gerber C 1994 Dielectric properties of sputtered $\mathrm{SrTiO}_{3}$ films Phys. Rev. B 4912095

[32] Sawaguchi E, Kikuchi A and Kodera Y 1962 Dielectric Constant of Strontium Titanate at Low Temperatures J. Phys. Soc. Jpn. 171666

[33] Lytle F W 1964 X-Ray Diffractometry of LowTemperature Phase Transformations in Strontium Titanate J. Appl. Phys. 352212

[34] Kaiser W and Zurek R 1966 Brillouin-and critical light scattering in SrTiO3-crystals Phys. Lett. 23 668-670

[35] Mikheev E, Raghavan S, Zhang J Y, Marshall P B, Kajdos A P, Balents L and Stemmer S 2016 Carrier density independent scattering rate in $\mathrm{SrTiO}_{3}$-based electron liquids Sci. Rep. 620865
[36]

van der Marel D, van Mechelen J L M and Mazin I I 2011 Common Fermi-liquid origin of $\mathrm{T}^{2}$ resistivity and superconductivity in n-type $\mathrm{SrTiO}_{3}$ Phys. Rev. $B \mathbf{8 4}$ 205111

[37] Klimin S N, Tempere J, van der Marel D and Devreese J T 2012 Microscopic mechanisms for the Fermi-liquid behavior of $\mathrm{Nb}$-doped strontium titanate Phys. Rev. B 86045113

[38] Cancellieri C, Mishchenko A S, Aschauer U, Filippetti A, Faber C, Barišić O S, Rogalev V A, Schmitt T, Nagaosa N and Strocov V N 2016 Polaronic metal state at the $\mathrm{LaAlO}_{3} / \mathrm{SrTiO}_{3}$ interface Nat. Commun. 7 10386

[39] Su S, Ho You J and Lee C 2013 Electron transport at interface of $\mathrm{LaAlO}_{3}$ and $\mathrm{SrTiO}_{3}$ band insulators J. Appl. Phys. 113093709

[40] Spinelli A, Torija M A, Liu C, Jan C and Leighton C 2010 Electronic transport in doped $\mathrm{SrTiO}_{3}$ : Conduction mechanisms and potential applications Phys. Rev. B 81155110

[41] Rice W D, Ambwani P, Bombeck M, Thompson J D, Haugstad G, Leighton C and Crooker S A 2014 Persistent optically induced magnetism in oxygendeficient strontium titanate Nat. Mater. 13 481-7

[42] Xu C, Bäumer C, Heinen R A, Hoffmann-Eifert S, Gunkel F and Dittmann R 2016 Disentanglement of growth dynamic and thermodynamic effects in $\mathrm{LaAlO}_{3} / \mathrm{SrTiO}_{3}$ heterostructures Sci. Rep. 622410

[43] Ambwani P, Xu P, Haugstad G, Jeong J S, Deng R, Mkhoyan K A, Jalan B and Leighton C 2016 Defects, stoichiometry, and electronic transport in $\mathrm{SrTiO}_{3-\delta}$ epilayers: A high pressure oxygen sputter deposition study J. Appl. Phys. 120055704

[44] Jalan B, Allen S J, Beltz G E, Moetakef P and Stemmer $\mathrm{S} 2011$ Enhancing the electron mobility of $\mathrm{SrTiO}_{3}$ with strain Appl. Phys. Lett. 98132102

[45] Kobayashi S, Mizumukai Y, Ohnishi T, Shibata N, Ikuhara Y and Yamamoto T 2015 High Electron Mobility of Nb-Doped $\mathrm{SrTiO}_{3}$ Films Stemming from Rod-Type Sr Vacancy Clusters ACS Nano 9 10769-77

[46] Janotti A, Steiauf D and Van de Walle C G 2011 Strain effects on the electronic structure of $\mathrm{SrTiO}_{3}$ : Toward high electron mobilities Phys. Rev. B 84201304 
[47] Lee H, Campbell N, Lee J, Asel T J, Paudel T R, Zhou $H$, Lee J W, Noesges B, Seo J, Park B, Brillson L J, Oh S H, Tsymbal E Y, Rzchowski M S and Eom C B 2018 Direct observation of a two-dimensional hole gas at oxide interfaces Nat. Mater. 17 231-6

[48] Chen $Y$ and Pryds N 2018 2D hole gas seen Nat. Mater. 17 215-6

[49] Chen Y Z, Trier F, Wijnands T, Green R J, Gauquelin $N$, Egoavil R, Christensen D V, Koster G, Huijben M, Bovet N, Macke S, He F, Sutarto R, Andersen N H, Sulpizio J A, Honig M, Prawiroatmodjo G E D K, Jespersen T S, Linderoth S, Ilani S, Verbeeck J, Van Tendeloo G, Rijnders G, Sawatzky G A and Pryds N 2015 Extreme mobility enhancement of twodimensional electron gases at oxide interfaces by charge-transfer-induced modulation doping Nat. Mater. 14 801-6

[50] Chen Y, Trier F, Kasama T, Christensen D V, Bovet N, Balogh Z I, Li H, Thydén K T S, Zhang W, Yazdi S, Norby P, Pryds N and Linderoth S 2015 Creation of High Mobility Two-Dimensional Electron Gases via Strain Induced Polarization at an Otherwise Nonpolar Complex Oxide Interface Nano Lett. 15 1849-54

[51] Huijben M, Koster G, Kruize M K, Wenderich S, Verbeeck J, Bals S, Slooten E, Shi B, Molegraaf H J A, Kleibeuker J E, van Aert S, Goedkoop J B, Brinkman A, Blank D H A, Golden M S, van Tendeloo G, Hilgenkamp H and Rijnders G 2013 Defect Engineering in Oxide Heterostructures by Enhanced Oxygen Surface Exchange Adv. Funct. Mater. 23 5240-8

[52] Son J, Moetakef P, Jalan B, Bierwagen O, Wright N J, Engel-Herbert R and Stemmer S 2010 Epitaxial $\mathrm{SrTiO}_{3}$ films with electron mobilities exceeding $30,000 \mathrm{~cm}^{2} \mathrm{~V}^{-}$ ${ }^{1} \mathrm{~s}^{-1}$ Nat. Mater. 9 482-4

[53] Matsubara Y, Takahashi K S, Bahramy M S, Kozuka Y, Maryenko D, Falson J, Tsukazaki A, Tokura Y and Kawasaki M 2016 Observation of the quantum Hall effect in $\delta$-doped $\mathrm{SrTiO}_{3}$ Nat. Commun. 711631

[54] Fête A, Cancellieri C, Li D, Stornaiuolo D, Caviglia A D, Gariglio S and Triscone J-M 2015 Growth-induced electron mobility enhancement at the $\mathrm{LaAlO}_{3} / \mathrm{SrTiO}_{3}$ interface Appl. Phys. Lett. 106051604

[55] Chen Y, Pryds N, Kleibeuker J E, Koster G, Sun J, Stamate E, Shen B, Rijnders G and Linderoth S 2011 Metallic and Insulating Interfaces of Amorphous
$\mathrm{SrTiO}_{3}$-Based Oxide Heterostructures Nano Lett. 11 3774-8

[56] Christensen D V, Frenkel Y, Schütz P, Trier F, Wissberg S, Claessen R, Kalisky B, Smith A, Chen Y Z and Pryds N 2018 Electron mobility in $\mathrm{\gamma}-\mathrm{Al}_{2} \mathrm{O}_{3} / \mathrm{SrTiO}_{3}$ Phys Rev Appl 9054004

[57] Christensen D V, von Soosten $M$, Trier F, Jespersen $T$ S, Smith A, Chen Y and Pryds N 2017 Controlling the carrier density of $\mathrm{SrTiO}_{3}$-based heterostructures with annealing Adv. Electron. Mater. 1700026

[58] Kozuka Y, Kim M, Ohta H, Hikita Y, Bell C and Hwang H Y 2010 Enhancing the electron mobility via deltadoping in $\mathrm{SrTiO}_{3}$ Appl. Phys. Lett. 97222115

[59] Jalan B, Stemmer S, Mack S and Allen S J 2010 Twodimensional electron gas in $\delta$-doped $\mathrm{SrTiO}_{3}$ Phys. Rev. B 82081103

[60] Herranz G, Basletić M, Bibes M, Carrétéro C, Tafra E, Jacquet $E$, Bouzehouane K, Deranlot $C$, Hamzić $A$, Broto J-M, Barthélémy A and Fert A 2007 High Mobility in $\mathrm{LaAlO}_{3} / \mathrm{SrTiO}_{3}$ Heterostructures: Origin, Dimensionality, and Perspectives Phys. Rev. Lett. 98 216803

[61] Kalabukhov A, Gunnarsson R, Börjesson J, Olsson E, Claeson T and Winkler D 2007 Effect of oxygen vacancies in the $\mathrm{SrTiO}_{3}$ substrate on the electrical properties of the $\mathrm{LaAlO}_{3} / \mathrm{SrTiO}_{3}$ interface Phys. Rev. B 75121404

[62] Yu L and Zunger A 2014 A polarity-induced defect mechanism for conductivity and magnetism at polarnonpolar oxide interfaces Nat. Commun. 55118

[63] Sanders T D, Gray M T, Wong F J and Suzuki Y 2015 $\mathrm{LaAlO}_{3} / \mathrm{SrTiO}_{3}$ interfaces doped with rare-earth ions Phys. Rev. B 91205112

[64] Xie Y, Bell C, Hikita Y, Harashima S and Hwang H Y 2013 Enhancing Electron Mobility at the LaAlO<sub $>3</$ sub/SrTiO 3 Interface by Surface Control Adv. Mater. 25 4735-8

[65] Trier F, Reich K V, Christensen D V, Zhang Y, Tuller H L, Chen Y, Shklovskii B I and Pryds N 2017 Universality of electron mobility in $\mathrm{LaAlO}_{3} / \mathrm{SrTiO}_{3}$ and bulk $\mathrm{SrTiO}_{3}$ Appl. Phys. Lett. 111092106

[66] Bell C, Harashima S, Kozuka Y, Kim M, Kim B G, Hikita Y and Hwang H Y 2009 Dominant Mobility 
Modulation by the Electric Field Effect at the $\mathrm{LaAlO}_{3} / \mathrm{SrTiO}_{3}$ Interface Phys. Rev. Lett. 103226802

[67] Zeng S, Lü W, Huang Z, Liu Z, Han K, Gopinadhan K, Li C, Guo R, Zhou W, Ma H H, Jian L, Venkatesan T and Ariando 2016 Liquid-Gated High Mobility and Quantum Oscillation of the Two-Dimensional Electron Gas at an Oxide Interface ACS Nano 10 4532-7

[68] Schütz P, Christensen D V, Borisov V, Pfaff F, Scheiderer P, Dudy L, Zapf M, Gabel J, Chen Y Z, Pryds N, Rogalev V A, Strocov V N, Lee T-L, Jeschke H O, Valentí R, Sing M and Claessen R 2017 Microscopic origin of the mobility enhancement at a spinel/perovskite oxide heterointerface revealed by photoemission spectroscopy Phys. Rev. B 96161409

[69] Nakagawa N, Hwang H Y and Muller D A 2006 Why some interfaces cannot be sharp Nat. Mater. 5 204-9

[70] Irvin P, Veazey J P, Cheng G, Lu S, Bark C-W, Ryu S, Eom C-B and Levy J 2013 Anomalous High Mobility in $\mathrm{LaAlO}_{3} / \mathrm{SrTiO}_{3}$ Nanowires Nano Lett. 13 364-8

[71] Janotti A and Van de Walle C G 2009 Fundamentals of zinc oxide as a semiconductor Rep. Prog. Phys. 72 126501

[72] Makino T, Segawa $Y$, Kawasaki $\mathrm{M}$ and Koinuma $\mathrm{H}$ 2005 Optical properties of excitons in $\mathrm{ZnO}$-based quantum well heterostructures Semicond. Sci. Technol. 20 S78-91

[73] Gopal P and Spaldin N A 2006 Polarization, piezoelectric constants, and elastic constants of $\mathrm{ZnO}$, $\mathrm{MgO}$, and CdO J. Electron. Mater. 35 538-542

[74] Button, K, Cohn D, Ortenbert M and Lax B 1972 Zeeman Splitting of Anomalous Shallow Bound States in ZnO Phys. Rev. Lett. 1637

[75] KISI E and ELCOMBE M 1989 u Parameters for the Wurtzite Structure of $\mathrm{ZnS}$ and $\mathrm{ZnO}$ using Powder Neutron Diffraction Acta Cryst 1867

[76] Wager J Transparent Electronics 2003

[77] Kawazoe H, Yasukawa M, Hyodo H, Kurita M, Yanagi $\mathrm{H}$ and Hosono $\mathrm{H} 1997 \mathrm{P}$-type electrical conduction in transparent thin films of CuAlO2 Nature 389 937-939

[78] Wei Shih C, Chin A, Fu Lu C and Fang Su W 2016 Remarkably high mobility ultra-thin-film metal-oxide transistor with strongly overlapped orbitals Sci. Rep.
[79] Nomura M, Ohta H, Takagi T, Hirano $\mathrm{M}$ and Hosono H 2004 Room-temperature fabrication of transparent flexible thin-film transistors using amorphous oxide semiconductors. Nature 2004 488-92

[80] Shih C W and Chin A 2017 Remarkably High Mobility Thin-Film Transistor on Flexible Substrate by Novel Passivation Material Sci. Rep. 7

[81] Look D C, Reynolds D C, Sizelove J R, Jones R L, Litton C W, Cantwell G and Harsch W C 1998 Electrical properties of bulk ZnO Solid State Commun. 105 399401

[82] Polyakov A Y, Smirnov N B, Govorkov A V, Kozhukhova E A, Pearton S J, Norton D P, Osinsky A and Dabiran A 2006 Electrical properties of undoped bulk ZnO substrates J. Electron. Mater. 35 663-669

[83] Udono H, Sumi Y, Yamada S and Kikuma I 2008 Crystal growth of ZnO bulk by CVT method using PVA J. Cryst. Growth 310 1827-31

[84] Cho M W, Harada C, Suzuki H, Minegishi T, Yao T, Ko $\mathrm{H}$, Maeda K and Nikura I 2005 Issues in ZnO homoepitaxy Superlattices Microstruct. 38 349-63

[85] Allen M W, Swartz C H, Myers T H, Veal T D, McConville C F and Durbin S M 2010 Bulk transport measurements in $\mathrm{ZnO}$ : The effect of surface electron layers Phys. Rev. B $\mathbf{8 1}$

[86] Yang X and Giles N C 2009 Hall effect analysis of bulk ZnO comparing different crystal growth techniques $J$. Appl. Phys. 105063709

[87] Bertazzi F, Bellotti E, Furno E and Goano M 2009 Experimental Electron Mobility in ZnO: A Reassessment Through Monte Carlo Simulation J. Electron. Mater. 38 1677-83

[88] Li X-H, Xu, JIN M, Shen $\mathrm{H}$ and Li X-M Electrical and optical properties of bulk $\mathrm{ZnO}$ single Crystal growth by Flux Bridgman Method 20063356

[89] McCluskey M D and Jokela S J 2009 Defects in ZnO J. Appl. Phys. 106071101

[90] Van de Walle C G 2000 Hydrogen as a cause of doping in zinc oxide Phys. Rev. Lett. 851012

[91] Lee E-C, Kim Y-S, Jin Y-G and Chang K J 2001 Compensation mechanism for $\mathrm{N}$ acceptors in $\mathrm{ZnO}$ Phys. Rev. B 64 
[92] Kohan A F, Ceder G, Morgan D and Van de Walle C G 2000 First-principles study of native point defects in ZnO Phys. Rev. B 6115019

[93] Fang Z-Q, Claflin B, Look D C, Kerr L L and Li X 2007 Electron and hole traps in $\mathrm{N}$-doped $\mathrm{ZnO}$ grown on $\mathrm{p}$ type Si by metalorganic chemical vapor deposition $J$. Appl. Phys. 102023714

[94] Yu Z G, Wu P and Gong H 2006 Control of $p$ - and ntype conductivities in $\mathrm{P}$ doped $\mathrm{ZnO}$ thin films by using radio-frequency sputtering Appl. Phys. Lett. 88 132114

[95] Sun J C, Zhao J Z, Liang H W, Bian J M, Hu L Z, Zhang H Q, Liang X P, Liu W F and Du G T 2007 Realization of ultraviolet electroluminescence from $\mathrm{ZnO}$ homojunction with n-ZnO/p-ZnO:As/GaAs structure Appl. Phys. Lett. 90121128

[96] Guo W, Allenic A, Chen Y B, Pan X Q, Che Y, Hu Z D and Liu $B 2007$ Microstructure and properties of epitaxial antimony-doped p-type $\mathrm{ZnO}$ films fabricated by pulsed laser deposition Appl. Phys. Lett. 90 242108

[97] Lu J G, Zhang Y Z, Ye Z Z, Zeng Y J, He H P, Zhu L P, Huang J Y, Wang L, Yuan J, Zhao B H and Li X H 2006 Control of $\mathrm{p}$ - and n-type conductivities in Li-doped ZnO thin films Appl. Phys. Lett. 89112113

[98] Krtschil A, Dadgar A, Oleynik N, Bläsing J, Diez A and Krost A 2005 Local p-type conductivity in zinc oxide dual-doped with nitrogen and arsenic Appl. Phys. Lett. 87262105

[99] Lu J G, Ye Z Z, Yuan G D, Zeng Y J, Zhuge F, Zhu L P, Zhao B H and Zhang S B 2006 Electrical characterization of $\mathrm{ZnO}$-based homojunctions Appl. Phys. Lett. 89053501

[100] Batzill M and Diebold U 2005 The surface and materials science of tin oxide Prog. Surf. Sci. 79 47154

[101] Vasheghani Farahani S K, Veal T D, King P D C, Zúniga-Pérez J, Munoz-Sanjosé $\mathrm{V}$ and McConville $\mathrm{C} F$ 2011 Electron mobility in CdO films J. Appl. Phys. 109 073712

[102] Ma N, Tanen N, Verma A, Guo Z, Luo T, Xing H (Grace) and Jena D 2016 Intrinsic electron mobility limits in $8-\mathrm{Ga}_{2} \mathrm{O}_{3}$ Appl. Phys. Lett. 109212101
[103] Look D C 2017 Mobility vs thickness in $n+-Z n O$ films: Effects of substrates and buffer layers Mater. Sci. Semicond. Process. 69 2-8

[104] Deokate R J, Bhosale C H and Rajpure K Y 2009 Synthesis and characterization of Cdln2O4 thin films by spray pyrolysis technique J. Alloys Compd. 473 L20-4

[105] Allali D, Bouhemadou A, Zerarga F, Ghebouli M A and Bin-Omran S 2012 Prediction study of the elastic and thermodynamic properties of the $\mathrm{SnMg2O} 4$, SnZn2O4 and SnCd2O4 spinel oxides Comput. Mater. Sci. 60 217-23

[106] Ellmer K 2012 Past achievements and future challenges in the development of optically transparent electrodes Nat. Photonics 6 809-17

[107] Berginski M, Hüpkes J, Reetz W, Rech B and Wuttig M 2008 Recent development on surface-textured ZnO:Al films prepared by sputtering for thin-film solar cell application Thin Solid Films 516 5836-41

[108] Iwata K, Sakemi T, Yamada A, Fons P, Awai K, Yamamoto T, Shirakata S, Matsubara K, Tampo H, Sakurai K, Ishizuka S and Niki S 2005 Improvement of ZnO TCO film growth for photovoltaic devices by reactive plasma deposition (RPD) Thin Solid Films 480-481 199-203

[109] Yamny S E and Rafea M A 2012 Preparation and Characterization of ZnO: In Transparent Conductor by Low Cost Dip Coating Technique J. Mod. Phys. 03 1060-9

[110] Sato M, English L Q, Hubbard B E and Sievers A J 2002 Influence of sample shape on the production of intrinsic localized modes in an antiferromagnetic lattice J. Appl. Phys. 91 8676-8678

[111] Leenheer A J, Perkins J D, van Hest M F A M, Berry J J, O'Hayre R P and Ginley D S 2008 General mobility and carrier concentration relationship in transparent amorphous indium zinc oxide films Phys. Rev. B 77

[112] Tsukazaki A, Yuji H, Akasaka S, Tamura K, Nakahara K, Tanabe T, Takasu H, Ohtomo A and Kawasaki M 2008 High Electron Mobility Exceeding $10^{4} \mathrm{~cm}^{2} \mathrm{~V}^{-1} \mathrm{~s}$ ${ }^{-1}$ in $\mathrm{Mg}_{x} \mathrm{Zn}_{1-x} \mathrm{O} / \mathrm{ZnO}$ Single Heterostructures Grown by Molecular Beam Epitaxy Appl. Phys. Express 1055004

[113] Tsukazaki A, Akasaka S, Nakahara K, Ohno Y, Ohno H, Maryenko D, Ohtomo A and Kawasaki M 2010 
Observation of the fractional quantum Hall effect in an oxide Nat. Mater. 9 889-93

[114] Kozuka Y, Tsukazaki A, Maryenko D, Falson J, Akasaka S, Nakahara K, Nakamura S, Awaji S, Ueno K and Kawasaki M 2011 Insulating phase of a twodimensional electron gas in $\mathrm{Mg} \times \mathrm{Zn} 1-\times \mathrm{O} / \mathrm{ZnO}$ heterostructures below $v=13$ Phys. Rev. $B 84$ 033304

[115] Falson J, Maryenko D, Kozuka Y, Tsukazaki A and Kawasaki M 2011 Magnesium Doping Controlled Density and Mobility of Two-Dimensional Electron Gas in Mg\$_\{x\}\$Zn\$_\{1-x\}\$O/ZnO Heterostructures Appl. Phys. Express 4091101

[116] Tampo H, Shibata H, Matsubara K, Yamada A, Fons $P$, Niki S, Yamagata $M$ and Kanie $H 2006$ Twodimensional electron gas in $\mathrm{Zn}$ polar $\mathrm{ZnMgO} / \mathrm{ZnO}$ heterostructures grown by radical source molecular beam epitaxy Appl. Phys. Lett. 89132113

[117] Nakano M, Tsukazaki A, Ohtomo A, Ueno K, Akasaka S, Yuji H, Nakahara K, Fukumura T and Kawasaki M 2010 Electronic-Field Control of Two-Dimensional Electrons in Polymer-Gated-Oxide Semiconductor Heterostructures Adv. Mater. 22 876-9

[118] Fonstad C G and Rediker R H 1971 Electrical Properties of High-Quality Stannic Oxide Crystals J. Appl. Phys. 42 2911-8

[119] Furubayashi Y, Hitosugi T, Yamamoto $Y$, Inaba K, Kinoda G, Hirose Y, Shimada T and Hasegawa T 2005 A transparent metal: Nb-doped anatase TiO2 Appl. Phys. Lett. 86252101

[120] Makino T, Segawa Y, Tsukazaki A, Ohtomo A and Kawasaki M 2005 Electron transport in $\mathrm{ZnO}$ thin films Appl. Phys. Lett. 87022101

[121] Rafique S, Han L, Neal A T, Mou S, Tadjer M J, French $\mathrm{R} \mathrm{H}$ and Zhao $\mathrm{H} 2016$ Heteroepitaxy of N-type $\beta$-Ga 2 $\mathrm{O}_{3}$ thin films on sapphire substrate by low pressure chemical vapor deposition Appl. Phys. Lett. 109 132103

[122] Koida T and Kondo M 2007 Comparative studies of transparent conductive $\mathrm{Ti}-, \mathrm{Zr}$-, and $\mathrm{Sn}$-doped In2O3 using a combinatorial approach J. Appl. Phys. 101 063713

[123] Liu C P, Foo Y, Kamruzzaman M, Ho C Y, Zapien J A, Zhu W, Li Y J, Walukiewicz W and Yu K M 2016 Effects of Free Carriers on the Optical Properties of Doped
CdO for Full-Spectrum Photovoltaics Phys. Rev. Appl. 6

[124] $\operatorname{Kim} H$ J, Kim U, Kim H M, Kim T H, Mun H S, Jeon BG, Hong K T, Lee W-J, Ju C, Kim K H and Char K 2012 High Mobility in a Stable Transparent Perovskite Oxide Appl. Phys. Express 5061102

[125] Braic L, Vasilantonakis N, Zou B, Maier S A, Alford N M, Zayats A V and Petrov P K 2015 Optimizing Strontium Ruthenate Thin Films for Near-Infrared Plasmonic Applications Sci. Rep. $\mathbf{5}$

[126] Mnatsakanov T T, Levinshtein M E, Pomortseva LI, Yurkov S N, Simin G S and Khan M A 2003 Carrier mobility model for GaN Solid-State Electron. 47 111115

[127] Rode D L and Knight S 1971 Electron transport in GaAs Phys. Rev. B 32534

[128] Wang H F, Liu Q Z, Chen F, Gao G Y, Wu W and Chen X H 2007 Transparent and conductive oxide films with the perovskite structure: La- and Sb-doped BaSnO3 J. Appl. Phys. 101106105

[129] Raghavan S, Schumann T, Kim H, Zhang J Y, Cain T A and Stemmer S 2016 High-mobility $\mathrm{BaSnO}_{3}$ grown by oxide molecular beam epitaxy APL Mater. 4016106

[130] Fujiwara K, Nishihara K, Shiogai J and Tsukazaki A 2017 Enhanced electron mobility at the twodimensional metallic surface of $\mathrm{BaSnO}_{3}$ electricdouble-layer transistor at low temperatures Appl. Phys. Lett. 110203503

[131] Lee W-J, Kim H J, Kang J, Jang D H, Kim T H, Lee J H and Kim K H 2017 Transparent Perovskite Barium Stannate with High Electron Mobility and Thermal Stability Annu. Rev. Mater. Res. 47 391-423

[132] Kornblum L, Faucher J, Morales-Acosta M D, Lee M L, Ahn C H and Walker F 2018 Oxide heterostructures for high density 2D electron gases on GaAs J. Appl. Phys. 123025302

[133] Zhang Y, Gan Y, Niu W, Norrman K, Yan X, Christensen D V, von Soosten M, Zhang $H$, Shen B, Pryds N, Sun J and Chen Y 2018 Tuning the TwoDimensional Electron Gas at Oxide Interfaces with TiO Configurations: Evidence from X-ray Photoelectron Spectroscopy ACS Appl. Mater. Interfaces 10 1434-9

[134] Zou K, Ismail-Beigi S, Kisslinger K, Shen X, Su D, Walker F J and Ahn C H 2015 LaTiO3/KTaO3 
interfaces: A new two-dimensional electron gas

system APL Mater. 3036104

[135] Paudel T R and Tsymbal E Y 2017 Prediction of a mobile two-dimensional electron gas at the LaSc 03

/ BaSn 03 (001) interface Phys. Rev. B 96

[136] Wang Y, Tang W, Cheng J, Nazir S and Yang K 2016

High-mobility two-dimensional electron gas in $\mathrm{SrGeO}$

3 - and $\mathrm{BaSnO}_{3}$-based perovskite oxide

heterostructures: an ab initio study Phys. Chem.

Chem. Phys. 18 31924-9 